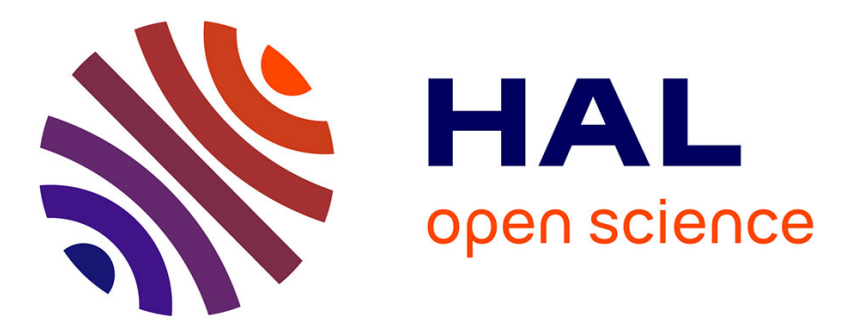

\title{
Optimal position targeting via decoupling fields
}

Stefan Ankirchner, Alexander Fromm, Thomas Kruse, Alexandre Popier

\section{To cite this version:}

Stefan Ankirchner, Alexander Fromm, Thomas Kruse, Alexandre Popier. Optimal position targeting via decoupling fields. 2018. hal-01500311v2

\section{HAL Id: hal-01500311 \\ https://hal.science/hal-01500311v2}

Preprint submitted on 16 Apr 2018

HAL is a multi-disciplinary open access archive for the deposit and dissemination of scientific research documents, whether they are published or not. The documents may come from teaching and research institutions in France or abroad, or from public or private research centers.
L'archive ouverte pluridisciplinaire HAL, est destinée au dépôt et à la diffusion de documents scientifiques de niveau recherche, publiés ou non, émanant des établissements d'enseignement et de recherche français ou étrangers, des laboratoires publics ou privés. 


\title{
Optimal position targeting via decoupling fields
}

\author{
Stefan Ankirchner*1, Alexander Fromm ${ }^{\dagger 1}$, Thomas Kruse ${ }^{\ddagger 2}$ and Alexandre Popier ${ }^{\S 3}$ \\ ${ }^{1}$ Institute for Mathematics, University of Jena, Ernst-Abbe-Platz 2, 07743 Jena, Germany \\ ${ }^{2}$ Faculty of Mathematics, University of Duisburg-Essen, Thea-Leymann-Str. 9, 45127 Essen, \\ Germany \\ ${ }^{3}$ Laboratoire Manceau de Mathématiques, Le Mans Université, Avenue O. Messiaen, 72085 Le Mans \\ cedex 9, France
}

April 16, 2018

\begin{abstract}
We consider a variant of the basic problem of the calculus of variations, where the Lagrangian is convex and subject to randomness adapted to a Brownian filtration. We solve the problem by reducing it, via a limiting argument, to an unconstrained control problem that consists in finding an absolutely continuous process minimizing the expected sum of the Lagrangian and the deviation of the terminal state from a given target position. Using the Pontryagin maximum principle we characterize a solution of the unconstrained control problem in terms of a fully coupled forward-backward stochastic differential equation (FBSDE). We use the method of decoupling fields for proving that the FBSDE has a unique solution. We exploit a monotonicity property of the decoupling field for solving the original constrained problem and characterize its solution in terms of an FBSDE with a free backward part.
\end{abstract}

2010 Mathematics Subject Classification. 49J05, 60G50, 60G99, 60H99, 93E20.

Keywords. Optimal stochastic control, calculus of variations, forward backward stochastic differential equation, decoupling field.

\section{Introduction}

The basic problem of the calculus of variations consists in minimizing an integral functional over a set of functions satisfying an initial and terminal condition. In this article we consider a version of the basic problem, where the Lagrangian is convex and subject to random influences supported by a Brownian motion $W$ on a probability space $(\Omega, \mathcal{F}, P)$. More precisely, let $T \in(0, \infty)$ and $f: \Omega \times[0, \infty) \times \mathbb{R}^{2} \rightarrow \mathbb{R}$ be a function, convex in the last two variables, such that for all $(x, a) \in \mathbb{R}^{2}$ the mapping $(\omega, t) \mapsto f(\omega, t, x, a)$ is progressively measurable

\footnotetext{
*s.ankirchner@uni-jena.de

†alexander.fromm@uni-jena.de

¥thomas.kruse@uni-due.de

§apopier@univ-lemans.fr
} 
with respect to $\left(\mathcal{F}_{t}\right)_{t \in[0, \infty)}$, the augmented filtration generated by the Brownian motion $W$. We show, under some additional analytic assumptions, existence of a solution of the following problem:

Minimize $\hat{J}(X)=\mathbb{E}\left[\int_{0}^{T} f\left(t, X_{t}, \dot{X}_{t}\right) d t\right]$ over all absolutely continuous

and progr. mb. processes $X$ satisfying $X_{0}=x_{0} \in \mathbb{R}$ and $X_{T}=0$.

We interpret $t$ as time, $X_{t}$ as the state and $\dot{X}_{t}$ as the velocity at time $t$.

Minimizing $\hat{J}(X)$ is a classical problem with many applications e.g. in physics, economics and engineering. We refer to the scripts of Gelfand and Fomin [12], Clarke [7] and Evans [9] for explicit applications and an overview on the deterministic version of the basic problem. Stochastic examples of problem (P) have been recently analyzed in the context of closing financial asset positions in illiquid markets (see e.g. the introduction in [15] for an overview). In these applications $f$ includes transaction costs, depending on the liquidation rate $\dot{X}$; moreover $f$ can incorporate measures of the risk exposure, depending on the volume $X_{t}$ of the remaining position.

In order to prove existence of a process $X$ minimizing the functional $\hat{J}(X)$ we study also a related control problem without the constraint $X_{T}=0$, but with an additional term in the cost functional penalizing any deviation of $X_{T}$ from zero. Let $g: \Omega \times \mathbb{R} \rightarrow \mathbb{R}$ be a function, convex in the second variable, such that for all $x \in \mathbb{R}$ the mapping $\omega \mapsto g(\omega, x)$ is $\mathcal{F}_{T}$-measurable. We solve, under some nice analytic assumptions, the following unconstrained control problem:

Minimize $J(X)=\mathbb{E}\left[\int_{0}^{T} f\left(t, X_{t}, \dot{X}_{t}\right) d t+g\left(X_{T}\right)\right]$ over all absolutely continuous

and progr. mb. processes $X$ satisfying $X_{0}=x_{0}$.

We show that by setting the penalty function equal to $g(x)=L x^{2}$ and letting $L \rightarrow \infty$ one can reduce the constrained problem (P) to the unconstrained one (UP).

By following a classic Bellman approach for solving (UP) (at least if $f$ and $g$ are deterministic functions), one obtains a non-linear Hamilton-Jacobi-Bellman (HJB) equation that is difficult to solve. By the Pontryagin maximum principle an optimal solution of (UP) can be characterized in terms of a forward-backward stochastic differential equation (FBSDE), where the forward component describes the optimal state dynamics and the backward component the dynamics of the so-called costate. The FBSDE for (UP) takes the form

$$
\begin{aligned}
X_{t} & =x-\int_{0}^{t} f_{y}^{*}\left(s, X_{s}, Y_{s}\right) \mathrm{d} s \\
Y_{t} & =g^{\prime}\left(X_{T}\right)-\int_{t}^{T} Z_{s} \mathrm{~d} W_{s}+\int_{t}^{T} f_{x}\left(s, X_{s}, f_{y}^{*}\left(s, X_{s}, Y_{s}\right)\right) \mathrm{d} s,
\end{aligned}
$$

where $f^{*}(t, x, \cdot)$ denotes the convex conjugate of the function $a \mapsto f(t, x, a), f_{y}^{*}$ its derivative w.r.t. $y$ and $f_{x}$ the derivative of $f$ w.r.t. $x$. Notice that the FBSDE (1) is fully coupled, i.e. the forward dynamics depend on the backward component $Y$, and the backward dynamics on the forward part $X$. It is a longstanding challenge to find conditions guaranteeing that a fully coupled FBSDE possesses a solution. Sufficient conditions are provided e.g. in [17], [23], [20], [24], [8], [18] (see also references therein). The method of decoupling fields, developped in [10] (see also the precursor articles [19], [11] and [18]), is practically useful for determining whether a solution exists. A decoupling field describes the functional dependence of the 
backward part $Y$ on the forward component $X$. If the coefficients of a fully coupled FBSDE satisfy a Lipschitz condition, then there exists a maximal non-vanishing interval possessing a solution triplet $(X, Y, Z)$ and a decoupling field with nice regularity properties. The method of decoupling fields consists in analyzing the dynamics of the decoupling field's gradient in order to determine whether the FBSDE has a solution on the whole time interval $[0, T]$.

We use decoupling fields since they provide an additional structure enabling to pass to the limit when the constant $L$ of the penalty function $g(x)=L x^{2}$ converges to infinity. Indeed, we show that the corresponding decoupling fields $u^{L}$ are non-decreasing in $L$. We can thus identify a limit $u^{\infty}$, which we further use for solving Problem $(\mathrm{P})$. In addition, from the convergence of $u^{L}$ we infer convergence of the corresponding solution processes $\left(X^{L}, Y^{L}, Z^{L}\right)$ to a process triplet $\left(X^{\infty}, Y^{\infty}, Z^{\infty}\right)$. We show that $\left(X^{\infty}, Y^{\infty}, Z^{\infty}\right)$ can be characterized as the unique solution of a pair of stochastic differential equations, where an initial and terminal condition is imposed on the forward equation, but no condition on the second (see (12) and Theorem 3.8). One can interpret the system as an FBSDE with a free backward component. To the best of our knowledge, this type of FBSDE is new since it cannot be reduced to the case studied in [29]. Moreover this FBSDE characterizes an optimal control for problem (P) as (1) does for problem (UP).

The FBSDE (1) can be shown to possess a solution by using the so-called continuation method, developed in $[14,28,24]$. In particular the problem (UP) has been solved already using this method (see e.g. [6, Section 5]). The continuation method, however, does not provide the existence of a decoupling field, which is fundamental in the present article for passing to the limit as the penalty converges to infinity and for solving Problem (P).

Solutions of problem (P) and (UP) have been obtained under some additional structural assumptions on the function $f$. One focus of the literature so far is set on cost functions $f$ that are additive. In [2] it is assumed that $f$ takes the form $f(t, x, a)=\gamma_{t}|x|^{p}+\eta_{t}|a|^{p}$, where $p>1$ and $(\eta, \gamma)$ is a pair of non-negative progressively measurable processes. The particular form allows to decouple the FBSDE (1), after a variable change. As the penality of any deviation of $X_{T}$ from 0 increases to infinity, the backward part of the decoupled FBSDE converges to a solution of a BSDE with singular terminal condition, a concept developed in [25], [26]. In the setting of the present article, the solution processes $(X, Y, Z)$ of (1) also converge as the penalty tends to infinity. The limiting processes turn out to be a solution of a coupled pair of stochastic differential equations, where an initial and terminal condition is imposed on the first equation, but no condition on the second (see Theorem 3.8).

Solving a fully coupled FBSDE is also not necessary in the case where $f$ is additive and linear-quadratic (see [3] and [4]), and even in some additive and polynomial cases with a Poisson measure as an additional source of randomness (see [13] and [16]).

The articles $[22,27]$ reformulate mass transportation problems as control problems imposing a constraint on the terminal state and hence bearing similarities with Problem $(\mathrm{P})$. In contrast to the present article, the position process in both articles is assumed to be disturbed by some Brownian noise; in [22] with constant and in [27] with freely controllable diffusion coefficient. In addition, [22] link the unique optimal control to an FBSDE related to the FBSDE (1). In contrast to our approach, the authors derive the FBSDE from the existence of an optimal control, but do not use it for proving existence in the first place.

The article is organized as follows. In Section 1 we specify some basic assumptions on the functions $f$ and $g$ under which we solve problem (UP) and (P) respectively. Moreover, we explain how to obtain a solution of (UP) from a solution $(X, Y, Z)$ of the $\operatorname{FBSDE}(1)$. 
Section 2.1 provides an overview of the method of decoupling fields. In Section 2.2 we apply the method to solve problem (UP). Finally, in Section 3 we solve problem (P) by reducing it to (UP).

\section{Problem formulation via FBSDEs}

Let $T \in(0, \infty)$ be a deterministic finite time horizon. Let $W=\left(W_{t}\right)_{t \in[0, T]}$ be a $d$-dimensional Brownian motion on a complete probability space $(\Omega, \mathcal{F}, \mathbb{P})$ and denote by $\left(\mathcal{F}_{t}\right)_{t \in[0, T]}$ the smallest filtration satisfying the usual conditions and containing the filtration generated by $W$.

Let $A \subseteq \mathbb{R}$ be a closed and connected set of possible control values satisfying inf $A \leq 0<$ $\sup A$. Let

$$
g: \Omega \times \mathbb{R} \rightarrow \mathbb{R}
$$

be measurable and

$$
f: \Omega \times[0, T] \times \mathbb{R} \times A \rightarrow \mathbb{R}
$$

be measurable such that for all $(x, a) \in \mathbb{R} \times A$ the mapping $(\omega, t) \mapsto f(\omega, t, x, a)$ is progressively measurable. We make the following additional assumptions on $f$ and $g$ :

(C0) For every fixed pair $(\omega, t) \in \Omega \times[0, T]$ the mappings $(x, a) \mapsto f(t, x, a)$ and $x \mapsto g(x)$ are convex ${ }^{1}$, with $f$ being strictly convex in $a$.

(C1) The mappings $A \ni a \mapsto f(t, x, a)$ and $\mathbb{R} \ni x \mapsto f(t, x, a)$ attain a minimum at zero (for all $\omega, t, x$ and all $\omega, t, a$ respectively). We also assume that $f(t, 0,0)=0$ for all $t \in[0, T]$. Observe that $f$ is then non-negative.

(C2) $f$ is coercive, i.e. there exist $p>1$ and $b>0$ such that

$$
\forall(\omega, t, x, a) \in \Omega \times[0, T] \times \mathbb{R} \times A: f(t, x, a) \geq b|a|^{p} .
$$

(C3) $g(\cdot)$ restricted to $[0, \infty)$ is twice continuously differentiable, $f(t, \cdot, \cdot)$ restricted to $[0, \infty) \times$ $(A \cap[0, \infty))$ is continuously differentiable, while $f_{x}(t, \cdot, \cdot)$ and $f_{a}(t, \cdot, \cdot)$ are continuously differentiable on $[0, \infty) \times A_{+}$, where $A_{+}:=A \cap(0, \infty)$. All second derivatives are bounded on compacts in $[0, \infty) \times A_{+}$and all first derivatives are bounded on compacts in $[0, \infty) \times(A \cap[0, \infty))$, uniformly in $(\omega, t)$.

(C4) The mapping $x \mapsto g(x)$ attains its minimum at zero (for all $\omega$ ). We also assume that $g(0)=0$. Observe that $g$ is then non-negative.

Remark 1.1. Note that the assumptions that $f(t, 0,0)=0$ for all $t \in[0, T]$ and $g(0)=0$ can be relaxed to the assumptions that $f(\cdot, 0,0) \in L^{1}(\Omega \times[0, T])$ and $g(0) \in L^{1}(\Omega)$. Indeed, in this case one can consider the problems (UP) and (P) with $\tilde{f}(t, x, a)=f(t, x, a)-f(t, 0,0)$ and $\tilde{g}(x)=g(x)-g(0)$ instead of $f$ and $g$ and add $\mathbb{E}\left[\int_{0}^{T} f(t, 0,0) d t+g(0)\right]$ outside the minimization problem.

\footnotetext{
${ }^{1}$ Note that here and in the sequel we follow the usual convention and omit the function argument $\omega$.
} 


\section{Problem (UP)}

For $(t, x) \in[0, T] \times \mathbb{R}$ we define $\mathcal{A}(t, x)$ as the set of all progressively measurable $\alpha: \Omega \times[t, T] \rightarrow$ $A$ such that a.s. $t \mapsto \alpha(\cdot, t)$ is integrable. Hence the process

$$
X_{s}^{t, x, \alpha}:=x-\int_{t}^{s} \alpha_{u} d u
$$

is well-defined for all $s \in[t, T]$. The dynamic version of problem (UP) then reads

$$
\text { Minimize } J(t, x, \alpha):=\mathbb{E}\left[\int_{t}^{T} f\left(s, X_{s}^{t, x, \alpha}, \alpha_{s}\right) d s+g\left(X_{T}^{t, x, \alpha}\right) \mid \mathcal{F}_{t}\right] \text { over all } \alpha \in \mathcal{A}(t, x) .
$$

The value function $v: \Omega \times[0, T] \times \mathbb{R} \rightarrow[0, \infty]$ of (3) is the random field that satisfies for all $(t, x) \in[0, T] \times \mathbb{R}$

$$
v(t, x)=\operatorname{essinf}_{\alpha \in \mathcal{A}(t, x)} J(t, x, \alpha) .
$$

For simplicity we sometimes write $X^{\alpha}$ or just $X$ instead of $X^{t, x, \alpha}$. So, for given $(t, x) \in$ $[0, T] \times \mathbb{R}$, the goal is to choose the control $\alpha$ from the set $\mathcal{A}(t, x)$ of admissible controls in such a way that $J$ is minimized.

The next result shows that when starting with a non-negative initial position, then it can not be optimal to choose $\alpha$ such that the position process is increasing or negative at some time point. This result is coherent with the absence of transaction-triggered price manipulation (see [1]). Note that for proving this statement we only need $(\mathrm{C} 0),(\mathrm{C} 1)$ and $(\mathrm{C} 4)$, but not (C2) and (C3).

Proposition 1.2. Let $(t, x) \in[0, T] \times[0, \infty)$. If $\alpha \in \mathcal{A}(t, x)$ is optimal in (3), then $X_{s}^{\alpha}=$ $x-\int_{t}^{s} \alpha_{r} d r, s \in[0, T]$, is non-increasing and non-negative. Moreover, for any $\alpha \in \mathcal{A}(t, x)$ there exists $\beta \in \mathcal{A}(t, x)$ such that $X^{\beta}$ is non-increasing and non-negative and $J(t, x, \beta) \leq J(t, x, \alpha)$.

The proof of Proposition 1.2 is provided in the appendix. Observe that by symmetry, when starting in a negative position, one can restrict the analysis to non-positive controls and positions, with straightforward adjustments in the hypothesis (C3) (differentiability condition for non positive values). In the following we consider only the positive case and always assume that any positions and controls are non-negative.

The so-called Hamiltonian of the control problem (3) is defined by

$$
\mathcal{H}(t, x, a, y):=-a y+f(t, x, a),
$$

for $t \in[0, T]$ and $(x, a, y) \in \mathbb{R} \times A \times \mathbb{R}$. Notice that

$$
\min _{a \in A} \mathcal{H}(t, x, a, y)=-f^{*}(t, x, y),
$$

where $f^{*}(t, x, \cdot)$ is the convex conjugate of $f(t, x, \cdot)$. Observe that condition (C2) guarantees that $f^{*}$ assumes real values only. For the following observation we need both $(\mathrm{C} 2)$ and $(\mathrm{C} 3)$.

Remark 1.3. For all $x, y \geq 0$ the following consideration holds: The minimum in (5) is attained at $a=f_{y}^{*}(t, x, y)$, where $f_{y}^{*}$ denotes the partial derivative of $f^{*}$ with respect to $y$. This partial derivative exists due to differentiability of $f$ w.r.t. the last parameter $a$ and (C2). More 
precisely, using Fermat's theorem applied to the minimization problem $\min _{a \in A} \mathcal{H}(t, x, a, y)=$ $\min _{a \in A_{+}} \mathcal{H}(t, x, a, y)$ one can deduce

$$
a=f_{y}^{*}(t, x, y)=f_{a}^{-1}(t, x, y), \quad \text { if } \quad y \in\left(f_{a}(t, x, 0), f_{a}\left(t, x, a_{\text {sup }}\right)\right),
$$

where $a_{\text {sup }}:=\sup A$ and where $f_{a}^{-1}(t, x, \cdot)$ denotes the inverse of the function $f_{a}(t, x, \cdot)$, which is strictly increasing.

Note that for $y \in\left[0, f_{a}(t, x, 0)\right]$ we have $f_{y}^{*}(t, x, y)=0$ and for $y \in\left[f_{a}\left(t, x, a_{\text {sup }}\right), \infty\right)$ we have $f_{y}^{*}(t, x, y)=a_{\text {sup }}$. This means that we can extend $f_{a}^{-1}(t, x, \cdot)$ to the whole of $[0, \infty)$ canonically, s.t. $f_{y}^{*}(t, x, y)=f_{a}^{-1}(t, x, y)$ holds everywhere and this extended $f_{a}^{-1}(t, x, \cdot)$ is still continuous and non-decreasing.

Finally notice the following straightforwardly verifiable properties of $f_{y}^{*}$ :

(i) $f_{y}^{*}(t, x, y) \geq 0$ for all $t \in[0, T]$ and $x, y \geq 0$,

(ii) $f_{y}^{*}(t, x, 0)=0$ for all $t \in[0, T]$ and $x \geq 0$,

(iii) $f_{y}^{*}(t, x, \cdot)$, which is defined on $[0, \infty)$, has $A \cap[0, \infty)$ as its range for all $t \in[0, T]$ and $x \geq 0$.

The following observation will be of importance later on:

Remark 1.4. Assume that $f_{a a}(t, x, a)>0$ holds for all $(\omega, t, x, a) \in \Omega \times[0, T] \times[0, \infty) \times A_{+}$ and that $f_{y}^{*}$ is weakly differentiable w.r.t. $(x, y)$ on $[0, \infty)^{2}$ (for the definition of the weak derivative see e.g. Section 2.1 in [32]). By applying the chain rule to the equation

$$
f_{a}\left(t, x, f_{y}^{*}(t, x, y)\right)=f_{a}(t, x, 0) \vee y \wedge f_{a}\left(t, x, a_{\mathrm{sup}}\right)
$$

we obtain

$$
\left(f_{y x}^{*}(t, x, y), f_{y y}^{*}(t, x, y)\right)=\left\{\begin{array}{l}
(0,0), \text { if } y \notin\left(f_{a}(t, x, 0), f_{a}\left(t, x, a_{\mathrm{sup}}\right)\right), \\
\left(-\frac{f_{a x}}{f_{a a}}\left(t, x, f_{y}^{*}(t, x, y)\right), \frac{1}{f_{a a}}\left(t, x, f_{y}^{*}(t, x, y)\right)\right), \text { otherwise }
\end{array}\right.
$$

We remark that in order to have $f_{a a}(t, x, a)>0$ for all $(x, a) \in[0, \infty) \times A_{+}$it is sufficient to require local Lipschitz continuity of $f_{y}^{*}(t, x, \cdot)$ for all $\omega, t$ and all $x \geq 0$. Indeed, for fixed $\omega, t, x$ the above formula for $f_{y y}^{*}(t, x, y)$ is satisfied on the set of all $y \geq 0$ satisfying $f_{a a}\left(t, x, f_{y}^{*}(t, x, y)\right)>0$ and this set cannot be empty (due to strict convexity). Due to the continuity of $f_{a a}\left(t, x, f_{y}^{*}(t, x, \cdot)\right)$ and the assumption of local boundedness of $f_{y y}^{*}(t, x, \cdot)$, the reciprocal value of $f_{a a}\left(t, x, f_{y}^{*}(t, x, \cdot)\right)$ must remain bounded on compact subsets of $[0, \infty)$. In particular $f_{a a}\left(t, x, f_{y}^{*}(t, x, y)\right)>0$ for all $y \geq 0$, which means $f_{a a}(t, x, a)>0$ for all $a \in A_{+}$.

Next we consider for $(t, x) \in[0, T] \times[0, \infty)$ the so-called adjoint forward-backward stochastic differential equation (FBSDE) for the control problem (3), given by

$$
\begin{aligned}
X_{s}^{t, x} & =x-\int_{t}^{s} f_{y}^{*}\left(r, X_{r}^{t, x}, Y_{r}^{t, x}\right) d r \\
Y_{s}^{t, x} & =g^{\prime}\left(X_{T}^{t, x}\right)-\int_{s}^{T} Z_{r}^{t, x} d W_{r}+\int_{s}^{T} f_{x}\left(r, X_{r}^{t, x}, f_{y}^{*}\left(r, X_{r}^{t, x}, Y_{r}^{t, x}\right)\right) d r
\end{aligned}
$$

for all $s \in[t, T]$. To simplify the notations, when there is no ambiguity, $\left(X^{t, x}, Y^{t, x}, Z^{t, x}\right)$ will be denoted by $(X, Y, Z)$. In this section and Sections 2.2 and 3 we mean by a solution to (7) a triplet $(X, Y, Z)=\left(X^{t, x}, Y^{t, x}, Z^{t, x}\right)$ of progressively measurable processes with values in $\mathbb{R} \times \mathbb{R} \times \mathbb{R}^{d}$ such that 
(i) $X$ and $Y$ are continuous and non-negative processes,

(ii) the processes $X, Y$ and $s \mapsto f_{y}^{*}\left(s, X_{s}, Y_{s}\right)$ are bounded and, finally,

(iii) the two equations (7) are satisfied a.s. for every fixed $s \in[t, T]$.

Note that under the above and due to (C3) the processes $s \mapsto f\left(s, X_{s}^{t, x}, f_{y}^{*}\left(s, X_{s}^{t, x}, Y_{s}^{t, x}\right)\right)$, $s \mapsto f_{x}\left(s, X_{s}^{t, x}, f_{y}^{*}\left(s, X_{s}^{t, x}, Y_{s}^{t, x}\right)\right)$, as well as the random variables $g\left(X_{T}^{t, x}\right)$ and $g^{\prime}\left(X_{T}^{t, x}\right)$ are also bounded. This implies that the stochastic integral $\int_{0}^{*} Z_{r}^{t, x} d W_{r}$ is a BMO martingale (see e.g. Proposition 1.1 in [5]). In particular for any $p \geq 1$ it holds that

$$
\mathbb{E}\left[\left(\int_{0}^{T}\left|Z_{r}^{t, x}\right|^{2} d r\right)^{p / 2}\right]<+\infty .
$$

Remark 1.5. We require that any solution satisfies $X, Y \geq 0$ to make sure that the equation (7) is well defined. Notice that we only assume that $g$ is differentiable on $[0, \infty)$ and that $f_{y}^{*}(t, x, y), f_{x}\left(t, x, f_{y}^{*}(t, x, y)\right)$ are defined for $x, y \geq 0$.

Constructing solutions to the above FBSDE is important for the following reason.

Proposition 1.6. Let $(t, x) \in[0, T] \times[0, \infty)$. If there exists a solution $\left(X^{t, x}, Y^{t, x}, Z^{t, x}\right)$ of (7), then the process $\alpha=\left(\alpha_{s}\right)_{s \in[t, T]}$ satisfying $\alpha_{s}=f_{y}^{*}\left(s, X_{s}^{t, x}, Y_{s}^{t, x}\right), s \in[t, T]$, is an optimal control for problem (3) with finite expected costs, i.e.

$$
v(t, x)=J(t, x, \alpha)<\infty .
$$

The proof of Proposition 1.6 is provided in the appendix.

\section{Problem (P)}

For all $(t, x) \in[0, T) \times \mathbb{R}$ let $\mathcal{A}^{0}(t, x):=\left\{\alpha \in \mathcal{A}(t, x) \mid X_{T}^{t, x, \alpha}=0\right.$ a.s. $\}$. The dynamic version of problem $(\mathrm{P})$ then reads

$$
\text { Minimize } \hat{J}(t, x, \alpha):=\mathbb{E}\left[\int_{t}^{T} f\left(s, X_{s}^{t, x, \alpha}, \alpha_{s}\right) d s \mid \mathcal{F}_{t}\right] \text { over all } \alpha \in \mathcal{A}^{0}(t, x) .
$$

The value function $\hat{v}: \Omega \times[0, T) \times \mathbb{R} \rightarrow[0, \infty]$ of $(9)$ is the random field that satisfies for all $(t, x) \in[0, T) \times \mathbb{R}$

$$
\hat{v}(t, x)=\operatorname{essinf}_{\alpha \in \mathcal{A}^{0}(t, x)} \hat{J}(t, x, \alpha) .
$$

In order to solve problem (9), we choose a penalization method (cf. [31, Section III.6] for problems with state constraint and [2] for a special case of Problem (P)). More precisely, we consider variants of problem (3) with penalty functions $g^{L}(x):=L x^{2}, x \in[0, \infty), L \in(0, \infty)$ and define

$$
J^{L}(t, x, \alpha):=\mathbb{E}\left[\int_{t}^{T} f\left(s, X_{s}, \alpha_{s}\right) d s+g^{L}\left(X_{T}\right) \mid \mathcal{F}_{t}\right]
$$

for $(t, x) \in[0, T] \times[0, \infty)$ and $\alpha \in \mathcal{A}(t, x)$. We show in Subsection 2.2, under some additional analytic conditions, that for all $(t, x) \in[0, T] \times[0, \infty)$ the $\operatorname{FBSDE}(7)$ associated to the problem of minimizing $J^{L}$ has a solution $\left(X^{L}, Y^{L}, Z^{L}\right)$. In Section 3 we prove that $\left(X^{L}, Y^{L}, Z^{L}\right)$ 
converges to a process $\left(X^{\infty}, Y^{\infty}, Z^{\infty}\right)$, as $L \rightarrow \infty$, that is characterized as the unique solution of the coupled SDEs:

$$
\begin{aligned}
& X_{s}^{\infty}=x-\int_{t}^{s} f_{y}^{*}\left(u, X_{u}^{\infty}, Y_{u}^{\infty}\right) d u, \quad X_{T}^{\infty}=\lim _{u \rightarrow T} X_{u}^{\infty}=0 \\
& Y_{s}^{\infty}=Y_{r}^{\infty}+\int_{s}^{r} f_{x}\left(u, X_{u}^{\infty}, f_{y}^{*}\left(u, X_{u}^{\infty}, Y_{u}^{\infty}\right)\right) d u-\int_{s}^{r} Z_{u}^{\infty} d W_{u},
\end{aligned}
$$

for all $s \in[t, T), r \in[s, T)$. Observe that in contrast to (7), the terminal position constraint translates here into a terminal condition on the first equation. On the second equation no boundary conditions are imposed. Moreover, we show in Section 3 that the optimal control $\alpha^{\infty}=f_{y}^{*}\left(\cdot, X^{\infty}, Y^{\infty}\right)$ belongs to $\mathcal{A}(t, x)$ and solves problem (9).

\section{Decoupling fields and problem (UP)}

\subsection{General results about the method of decoupling fields}

As mentioned above, solving (7) is crucial in constructing optimal controls. In Section 2.2 the solvability of (7) under the assumption that the functions $f_{y}^{*}, g^{\prime}$ and $f_{x}$ are uniformly Lipschitz continuous in $(x, y) \in[0, \infty)^{2}$ and are zero if we plug in the special value $(x, y)=(0,0)$.

Note that even under these Lipschitz assumptions, it is not trivial to show well-posedness of (7) due to its coupled nature. It is necessary to take more subtle structural properties into account to conduct the proof. Our arguments are based on the so-called method of decoupling fields which we briefly summarize in this section. Owing to their general significance, we treat the theory of FBSDEs and their decoupling fields in a more general framework than might be needed for investigating well-posedness of (7).

Let $T>0$ be a fixed finite time horizon and $\left(\Omega, \mathcal{F},\left(\mathcal{F}_{t}\right)_{t \in[0, T]}, \mathbb{P}\right)$ a complete filtered probability space, where $\mathcal{F}_{0}$ consists of all null sets, $\left(W_{t}\right)_{t \in[0, T]}$ is a $d$-dimensional Brownian motion and $\mathcal{F}_{t}:=\sigma\left(\mathcal{F}_{0},\left(W_{s}\right)_{s \in[0, t]}\right)$ with $\mathcal{F}:=\mathcal{F}_{T}$. For $t \in[0, T], x \in \mathbb{R}^{n}$ we consider an FBSDE of the form

$$
\begin{aligned}
& X_{s}^{t, x}=x+\int_{t}^{s} \mu\left(r, X_{r}^{t, x}, Y_{r}^{t, x}\right) d r \\
& Y_{s}^{t, x}=\xi\left(X_{T}^{t, x}\right)+\int_{s}^{T} \phi\left(r, X_{r}^{t, x}, Y_{r}^{t, x}\right) d r-\int_{s}^{T} Z_{r}^{t, x} d W_{r},
\end{aligned}
$$

for $0 \leq t \leq s \leq T$, where $n, m \in \mathbb{N}_{>0}$ and $\xi: \Omega \times \mathbb{R}^{n} \rightarrow \mathbb{R}^{m}, \mu:[0, T] \times \Omega \times \mathbb{R}^{n} \times \mathbb{R}^{m} \rightarrow \mathbb{R}^{n}$, $\phi:[0, T] \times \Omega \times \mathbb{R}^{n} \times \mathbb{R}^{m} \rightarrow \mathbb{R}^{m}$ are measurable functions. Throughout the whole section $\mu$ and $\phi$ are assumed to be progressively measurable with respect to $\left(\mathcal{F}_{t}\right)_{t \in[0, T]}$ and the random variable $\xi(x)$ is $\mathcal{F}_{T}$-measurable for all $x \in \mathbb{R}^{n}$. The FBSDE (13) is a generalization of the FBSDE (7).

Remark 2.1. Note that the decoupling field theory has been developed in a much more general setting, where $\mu$ and $\phi$ can also depend on $Z$ and/or where a diffusion part can be added in the forward equation. See $[11,10,18]$ for more details.

A decoupling field comes with an richer structure than just a solution $(X, Y, Z)$.

Definition 2.2. Let $t \in[0, T]$. A function $u:[t, T] \times \Omega \times \mathbb{R}^{n} \rightarrow \mathbb{R}^{m}$ with $u(T, \cdot)=\xi$ a.e. is called decoupling field for $(\xi,(\mu, \phi))$ on $[t, T]$ if for all $t_{1}, t_{2} \in[t, T]$ with $t_{1} \leq t_{2}$ and any $\mathcal{F}_{t_{1}}{ }^{-}$ measurable $X_{t_{1}}: \Omega \rightarrow \mathbb{R}^{n}$ there exist progressively measurable processes $(X, Y, Z)$ on $\left[t_{1}, t_{2}\right]$ 
such that

$$
\begin{aligned}
X_{s} & =X_{t_{1}}+\int_{t_{1}}^{s} \mu\left(r, X_{r}, Y_{r}\right) d r, \\
Y_{s} & =Y_{t_{2}}+\int_{s}^{t_{2}} \phi\left(r, X_{r}, Y_{r}\right) d r-\int_{s}^{t_{2}} Z_{r} d W_{r}, \\
Y_{s} & =u\left(s, X_{s}\right),
\end{aligned}
$$

for all $s \in\left[t_{1}, t_{2}\right]$. In particular, we want all integrals to be well-defined.

Some remarks about this definition are in place.

- The first equation in (14) is called the forward equation, the second the backward equation and the third will be referred to as the decoupling condition.

- Note that, if $t_{2}=T$, we get $Y_{T}=\xi\left(X_{T}\right)$ a.s. as a consequence of the decoupling condition together with $u(T, \cdot)=\xi$. At the same time $Y_{T}=\xi\left(X_{T}\right)$ together with decoupling condition implies $u(T, \cdot)=\xi$ a.e.

- If $t_{2}=T$ we can say that a triplet $(X, Y, Z)$ solves the FBSDE starting at time $t_{1}$, meaning that it satisfies the forward and the backward equation, together with $Y_{T}=$ $\xi\left(X_{T}\right)$. This relationship $Y_{T}=\xi\left(X_{T}\right)$ is referred to as the terminal condition.

Decoupling random fields can be pasted together, a useful property that has been already observed from the link to quasilinear PDEs (see e.g. [8, 17, 18, 21]):

Lemma 2.3 ([10], Lemma 2.1.2). Let u be a decoupling field for $(\xi,(\mu, \phi))$ on $[t, T]$ and $\tilde{u}$ be a decoupling field for $(u(t, \cdot),(\mu, \phi))$ on $[s, t]$, for $0 \leq s<t<T$. Then, the map $\hat{u}$ given by $\hat{u}:=\tilde{u} \mathbf{1}_{[s, t]}+u \mathbf{1}_{(t, T]}$ is a decoupling field for $(\xi,(\mu, \phi))$ on $[s, T]$.

We want to remark that, if $u$ is a decoupling field and $\tilde{u}$ is a modification of $u$, i.e. for each $s \in[t, T]$ the functions $u(s, \omega, \cdot)$ and $\tilde{u}(s, \omega, \cdot)$ coincide for almost all $\omega \in \Omega$, then $\tilde{u}$ is also a decoupling field to the same problem. Hence, $u$ could also be referred to as a class of modifications and a progressively measurable and in some sense right-continuous representative exists if the decoupling field is Lipschitz continuous in $x$ (Lemma 2.1.3 in [10]).

For the following we need to fix briefly further notation.

Let $I \subseteq[0, T]$ be an interval and $u: I \times \Omega \times \mathbb{R}^{n} \rightarrow \mathbb{R}^{m}$ a map such that $u(s, \cdot)$ is measurable for every $s \in I$. We define

$\mathfrak{L}_{u, x}:=\sup _{s \in I} \inf \left\{\mathfrak{L} \geq 0 \mid\right.$ for a.a. $\omega \in \Omega:\left|u(s, \omega, x)-u\left(s, \omega, x^{\prime}\right)\right| \leq \mathfrak{L}\left|x-x^{\prime}\right|$ for all $\left.x, x^{\prime} \in \mathbb{R}^{n}\right\}$,

where $\inf \emptyset:=\infty$. We also set $\mathfrak{L}_{u, x}:=\infty$ if $u(s, \cdot)$ is not measurable for every $s \in I$. One can show that $\mathfrak{L}_{u, x}<\infty$ is equivalent to $u$ having a modification which is truly Lipschitz continuous in $x \in \mathbb{R}^{n}$.

For an integrable real valued random variable $F$ the expression $\mathbb{E}_{t}[F]$ refers to $\mathbb{E}\left[F \mid \mathcal{F}_{t}\right]$, while $\mathbb{E}_{t, \infty}[F]$ refers to ess $\sup \mathbb{E}\left[F \mid \mathcal{F}_{t}\right]$, which might be $\infty$, but is always well defined as the infimum of all constants $c \in[-\infty, \infty]$ such that $\mathbb{E}\left[F \mid \mathcal{F}_{t}\right] \leq c$ a.s. Additionally, we write $\|F\|_{\infty}$ for the essential supremum of $|F|$.

Finally for a matrix $A \in \mathbb{R}^{N \times n}$ and a vector $v \in S^{n-1}:=\left\{x \in \mathbb{R}^{n}:|x|=1\right\}$, we define $|A|_{v}:=|A v|$ as the norm of $A$ in the direction $v$. 
In practice it is important to have explicit knowledge about the regularity of $(X, Y, Z)$. For instance, it is important to know in which spaces the processes live, and how they react to changes in the initial value.

Definition 2.4. Let $u$ : $[t, T] \times \Omega \times \mathbb{R}^{n} \rightarrow \mathbb{R}^{m}$ be a decoupling field to $(\xi,(\mu, \phi))$.

1. We say $u$ to be weakly regular if $\mathfrak{L}_{u, x}<\infty$ and $\sup _{s \in[t, T]}\|u(s, \cdot, 0)\|_{\infty}<\infty$.

2. A weakly regular decoupling field $u$ is called strongly regular if for all fixed $t_{1}, t_{2} \in[t, T]$, $t_{1} \leq t_{2}$, the processes $(X, Y, Z)$ arising in (14) are a.e unique and satisfy

$$
\sup _{s \in\left[t_{1}, t_{2}\right]} \mathbb{E}_{t_{1}, \infty}\left[\left|X_{s}\right|^{2}\right]+\sup _{s \in\left[t_{1}, t_{2}\right]} \mathbb{E}_{t_{1}, \infty}\left[\left|Y_{s}\right|^{2}\right]+\mathbb{E}_{t_{1}, \infty}\left[\int_{t_{1}}^{t_{2}}\left|Z_{s}\right|^{2} d s\right]<\infty
$$

for each constant initial value $X_{t_{1}}=x \in \mathbb{R}^{n}$. In addition they are required to be measurable as functions of $(x, s, \omega)$ and even weakly differentiable w.r.t. $x \in \mathbb{R}^{n}$ such that for every $s \in\left[t_{1}, t_{2}\right]$ the mappings $X_{s}$ and $Y_{s}$ are measurable functions of $(x, \omega)$ and even weakly differentiable w.r.t. $x$ such that

$$
\begin{aligned}
& \operatorname{ess} \sup _{x \in \mathbb{R}^{n}} \sup _{v \in S^{n-1}} \sup _{s \in\left[t_{1}, t_{2}\right]} \mathbb{E}_{t_{1}, \infty}\left[\left|\frac{\mathrm{d}}{\mathrm{d} x} X_{s}\right|_{v}^{2}\right]<\infty, \\
& \operatorname{ess}_{\sup } \sup _{x \in \mathbb{R}^{n}} \sup _{v \in S^{n-1}} \mathbb{E}_{s \in\left[t_{1}, t_{2}\right]} \mathbb{E}_{t_{1}, \infty}\left[\left|\frac{\mathrm{d}}{\mathrm{d} x} Y_{s}\right|_{v}^{2}\right]<\infty, \\
& \operatorname{ess~sup}_{x \in \mathbb{R}^{n}} \sup _{v \in S^{n-1}} \mathbb{E}_{t_{1}, \infty}\left[\int_{t_{1}}^{t_{2}}\left|\frac{\mathrm{d}}{\mathrm{d} x} Z_{s}\right|_{v}^{2} \mathrm{~d} s\right]<\infty \text {. }
\end{aligned}
$$

3. We say that a decoupling field on $[t, T]$ is strongly regular on a subinterval $\left[t_{1}, t_{2}\right] \subseteq[t, T]$ if $u$ restricted to $\left[t_{1}, t_{2}\right]$ is a strongly regular decoupling field for $\left(u\left(t_{2}, \cdot\right),(\mu, \phi)\right)$.

Under suitable conditions a rich existence, uniqueness and regularity theory for decoupling fields can be developed. The basis of the theory is Theorem 2.5 below, which is proven in Chapter 2 of [10].

Assumption (SLC): $(\xi,(\mu, \phi))$ satisfies standard Lipschitz conditions (SLC) if

1. $(\mu, \phi)$ are Lipschitz continuous in $(x, y)$ with Lipschitz constant $\mathfrak{L}$,

2. $\|(|\mu|+|\phi|)(\cdot, \cdot, 0,0)\|_{\infty}<\infty$,

3. $\xi: \Omega \times \mathbb{R} \rightarrow \mathbb{R}$ is measurable such that $\|\xi(\cdot, 0)\|_{\infty}<\infty$ and $\mathfrak{L}_{\xi, x}<\infty$.

Theorem 2.5 ([10], Theorem 2.2.1). Suppose $(\xi,(\mu, \phi))$ satisfies (SLC). Then there exists a time $t \in[0, T)$ such that $(\xi,(\mu, \phi))$ has a unique (up to modification) decoupling field $u$ on $[t, T]$ with $\mathfrak{L}_{u, x}<\infty$ and $\sup _{s \in[t, T]}\|u(s, \cdot, 0)\|_{\infty}<\infty$.

This local theory for decoupling fields can be systematically extended to global results based on fairly simple "small interval induction" arguments (Lemmas 2.5.1 and 2.5.2 in [10]).

Theorem 2.6 ([10], Corollaries 2.5.3, 2.5.4 and 2.5.5). Suppose that $(\xi,(\mu, \phi))$ satisfies (SLC). 
1. Global uniqueness: If there are two weakly regular decoupling fields $u^{(1)}, u^{(2)}$ to the corresponding problem on some interval $[t, T]$, then we have $u^{(1)}=u^{(2)}$ up to modifications.

2. Global regularity: If there exists a weakly regular decoupling field u to this problem on some interval $[t, T]$, then $u$ is strongly regular.

3. If there exists a weakly regular decoupling field $u$ of the corresponding FBSDE on some interval $[t, T]$, then for any initial condition $X_{t}=x \in \mathbb{R}$ there is a unique solution $(X, Y, Z)$ of the FBSDE on $[t, T]$ satisfying

$$
\sup _{s \in[t, T]} \mathbb{E}\left[\left|X_{s}\right|^{2}\right]+\sup _{s \in[t, T]} \mathbb{E}\left[\left|Y_{s}\right|^{2}\right]+\mathbb{E}\left[\int_{t}^{T}\left|Z_{s}\right|^{2} d s\right]<\infty .
$$

In order to have a notion of global existence we need the following definition:

Definition 2.7. We define the maximal interval $I_{\max } \subseteq[0, T]$ of the problem given by $(\xi,(\mu, \phi))$ as the union of all intervals $[t, T] \subseteq[0, T]$, such that there exists a weakly regular decoupling field $u$ on $[t, T]$.

Note that the maximal interval might be open to the left. Also, let us remark that we define a decoupling field on such an interval as a mapping which is a decoupling field on every compact subinterval containing $T$. Similarly we can define weakly and strongly regular decoupling fields as mappings which restricted to an arbitrary compact subinterval containing $T$ are weakly (or strongly) regular decoupling fields in the sense of the definitions given above.

Finally, we have global existence and uniqueness on the maximal interval:

Theorem 2.8 (Global existence in weak form, [10], Theorem 5.1.11 and Lemma 5.1.12). Let $(\xi,(\mu, \phi))$ satisfy (SLC). Then there exists a unique weakly regular decoupling field $u$ on $I_{\max }$. This $u$ is even strongly regular. Furthermore, either $I_{\max }=[0, T]$ or $I_{\max }=\left(t_{\min }, T\right]$, where $0 \leq t_{\min }<T$. In the latter case we have

$$
\lim _{t \downarrow t_{\text {min }}} \mathfrak{L}_{u(t, \cdot), x}=\infty .
$$

Note that in particular cases the last statement allows to show "strong global existence", i.e. $I_{\max }=[0, T]$, via contradiction and, thereby, is the basis of the so-called method of decoupling fields. We employ this idea in the section.

\subsection{Solving problem (UP)}

In this section we provide sufficient conditions for the FBSDE (7) to possess a unique solution on $[0, T]$. With Proposition 1.6 we then immediately obtain a solution of problem (UP).

In addition to conditions $(\mathrm{C} 0)$ to $(\mathrm{C} 4)$ we assume throughout this subsection the following assumptions.

(D1) The functions $(x, y) \mapsto f_{y}^{*}(t, x, y),(x, y) \mapsto f_{x}\left(t, x, f_{y}^{*}(t, x, y)\right)$ and $x \mapsto g^{\prime}(x)$ are Lipschitz continuous on $[0, \infty) \times[0, \infty)$ and $[0, \infty)$ respectively, uniformly in $(\omega, t) \in$ $\Omega \times[0, T]$.

(D2) It holds that $g^{\prime}(0)=f_{x}(t, 0,0)=0$ for all $(\omega, t) \in \Omega \times[0, T]$. 
We give an example of a generator satisfying (D1) and (D2) in Example 3.7 below. Notice that in general the functions $f_{y}^{*}(t, \cdot, \cdot), g^{\prime}, f_{x}\left(t, \cdot, f_{y}^{*}(t, \cdot, \cdot)\right)$ are defined only on $[0, \infty) \times[0, \infty)$, i.e. for non-negative $x, y$. We extend them to the whole of $\mathbb{R}^{2}$ by projecting $(x, y)$ to $(x \vee 0, y \vee 0)$ and plugging the projected value in the respective function. Note that condition (D1) is still maintained after this extension. By a slight abuse of notation we denote the new functions again by $f_{y}^{*}(t, \cdot, \cdot), g^{\prime}, f_{x}\left(t, \cdot, f_{y}^{*}(t, \cdot, \cdot)\right)$. Note that (D1) implies that these three functions are weakly differentiable on the whole of $\mathbb{R}^{2}$. Furthermore the weak derivatives w.r.t. $x$ vanish whenever $x \leq 0$ and the weak derivatives w.r.t. $y$ vanish whenever $y \leq 0$.

Now the parameter functions of the problem (7) satisfy (SLC), such that the theory of decoupling fields can be applied. We next show that the problem is well-posed and can be solved on the whole of $[0, T]$. We start with an auxiliary result stating that if the initial condition is positive, then the solution process of the forward equation remains always positive.

Lemma 2.9. Let $u$ be a weakly regular decoupling field on $[t, T]$ such that $u(s, 0)=0$ for all $s \in[t, T]$. Let $x \in(0, \infty)$ and $(X, Y, Z)$ be the solution of FBSDE (7) on $[t, T]$ with initial condition $X_{t}=x>0$. Then it holds that $X_{s}>0$ for all $s \in[t, T]$.

Proof. The forward equation of (7) together with the decoupling condition imply that for all $s \in[t, T]$ it holds

$$
X_{s}=x-\int_{t}^{s} f_{y}^{*}\left(r, X_{r}, u\left(r, X_{r}\right)\right) d r
$$

We have $f_{y}^{*}(r, 0,0)=u(r, 0)=0$ for all $r \in[t, T]$ and, therefore, for all $r \in[t, T]$

$$
\left|f_{y}^{*}\left(r, X_{r}, u\left(r, X_{r}\right)\right)\right| \leq \mathfrak{L}_{f_{y}^{*}}\left(\left|X_{r}\right|+\mathfrak{L}_{u} \cdot\left|X_{r}\right|\right)=\mathfrak{L}_{f_{y}^{*}}\left(1+\mathfrak{L}_{u}\right)\left|X_{r}\right|,
$$

where $\mathfrak{L}_{f_{y}^{*}}$ is a Lipschitz constant of $f_{y}^{*}$ w.r.t. the last two components $(x, y)$ and $L_{u}$ is a Lipschitz constant of $u$ w.r.t. $x$. Let $\theta:[t, T] \rightarrow \mathbb{R}$ be the unique solution to the ODE

$$
\theta_{s}=x-\int_{t}^{s} \mathfrak{L}_{f_{y}^{*}}\left(1+\mathfrak{L}_{u}\right)\left|\theta_{r}\right| d r, \quad s \in[t, T] .
$$

It is straightforward to verify that $\theta_{s}=x \cdot \exp \left(-\mathfrak{L}_{f_{y}^{*}}\left(1+\mathfrak{L}_{u}\right)(s-t)\right), s \in[t, T]$, is the solution. A comparison principle for ODEs implies that $X \geq \theta>0$.

Theorem 2.10. The maximal interval associated with $F B S D E(7)$ satisfies $I_{\max }=[0, T]$. Furthermore, the unique weakly regular decoupling field $u$ on $[0, T]$ satisfies $u(t, x)=0$ for all $x \leq 0$ and $t \in[0, T]$.

Proof. Suppose $I_{\max }=[0, T]$ does not hold. Then $I_{\max }=\left(t_{\min }, T\right]$, where $t_{\min } \in[0, T)$. We show that this leads to a contradiction. Let $u$ be the unique weakly regular decoupling field on $\left(t_{\min }, T\right]$. We next show that there exists a uniform bound on $u_{x}$. In the following let $t_{0} \in\left(t_{\min }, T\right]$ and $x \in \mathbb{R}$ be arbitrary. Let $(X, Y, Z)$ be the solution of the corresponding FBSDE (7) on $\left[t_{0}, T\right]$ with initial condition $X_{t_{0}}=x$. We define the processes

$$
D_{t}=\frac{\mathrm{d} X_{t}}{\mathrm{~d} x}, \quad C_{t}=\frac{\mathrm{d} Y_{t}}{\mathrm{~d} x} \quad \text { and } \quad \Psi_{t}=u_{x}\left(t, X_{t}\right), \quad t \in\left(t_{0}, T\right] .
$$

These objects are well defined due to strong regularity of $u$.

First, let us consider the case $x \leq 0$. It is straightforward to verify that $X, Y$ are both constant: $X=x$ and $Y=0$. This is due to uniqueness and the fact that these constant 
processes together with $Z=0$ solve the FBSDE as $g^{\prime}$ vanishes for non-positive values. This means that $u\left(t_{0}, x\right)=0$ for $x \leq 0$, which implies $u_{x}\left(t_{0}, x\right)=0$ for $x \leq 0$.

Second, let us assume $x>0$. Note that Lemma 2.9 implies that $X_{t}>0$, a.s. for all $t \in\left[t_{0}, T\right]$. This further entails $Y_{t} \geq 0$, a.s. Indeed, note that $Y$ satisfies for all $t \in\left[t_{0}, T\right]$

$$
Y_{t}=\mathbb{E}\left[g^{\prime}\left(X_{T}\right)+\int_{t}^{T} f_{x}\left(r, X_{r}, f_{y}^{*}\left(r, X_{r}, Y_{r}\right)\right) \mathrm{d} r \mid \mathcal{F}_{t}\right]
$$

Since $g^{\prime}$ and $f_{x}$ are non-negative we have $Y_{t} \geq 0$, a.s. for all $t \in\left[t_{0}, T\right]$.

By differentiating the forward equation w.r.t. the intial value $x \in \mathbb{R}$ one can show that $D$ satisfies the SDE

$$
d D_{t}=-\left(f_{y x}^{*}\left(t, X_{t}, Y_{t}\right) D_{t}+f_{y y}^{*}\left(t, X_{t}, Y_{t}\right) C_{t}\right) d t,
$$

with initial value $D_{t_{0}}=1$. Notice that $f_{y}^{*}$ is not necessarily differentiable in a point $(x, y)$ if $y \in\left\{f_{a}(t, x, 0), f_{a}\left(t, x, a_{\text {sup }}\right)\right\}$. The function $f_{y}^{*}$ is, however, weakly differentiable. Throughout the proof we define $f_{y x}^{*}(t, x, y)=f_{y y}^{*}(t, x, y)=0$ whenever $y \in\left\{f_{a}(t, x, 0), f_{a}\left(t, x, a_{\text {sup }}\right)\right\}$ (in other words, we define $\left(f_{y x}^{*}, f_{y y}^{*}\right)$ as in Remark 1.4). The chain rule for weak derivatives allows to differentiate inside the integral in the forward equation (see e.g. Lemma A.2.5. of [10] and Lemma A.3.1. of [10]).

We now show that $D$ remains positive on the whole interval $\left[t_{0}, T\right]$. To this end let $\tau \in\left[t_{0}, T\right]$ be a stopping time such that $D$ is positive on $\left[t_{0}, \tau\right]$. Then we have $\Psi_{t}=C_{t} D_{t}^{-1}$ on $\left[t_{0}, \tau\right]$. Note that

$$
\begin{aligned}
d D_{t}^{-1} & =\frac{f_{y x}^{*}\left(t, X_{t}, Y_{t}\right) D_{t}+f_{y y}^{*}\left(t, X_{t}, Y_{t}\right) C_{t}}{D_{t}^{2}} d t \\
& =D_{t}^{-1}\left(f_{y x}^{*}\left(t, X_{t}, Y_{t}\right)+f_{y y}^{*}\left(t, X_{t}, Y_{t}\right) \Psi_{t}\right) d t
\end{aligned}
$$

on the stochastic interval $\left[t_{0}, \tau\right]$. Consequently, it holds for all $t \in\left[t_{0}, T\right]$

$$
D_{t}^{-1}=\exp \left(\int_{t_{0}}^{t} f_{y x}^{*}\left(s, X_{s}, Y_{s}\right)+f_{y y}^{*}\left(s, X_{s}, Y_{s}\right) \Psi_{s} d s\right)
$$

Since the decoupling field is weakly regular, $u_{x}$ and hence $\Psi$ are bounded on $\left[t_{0}, T\right]$. Moreover, $f_{y x}^{*}, f_{y y}^{*}$ are uniformly bounded. Therefore, $D^{-1}$ is bounded on $\left[t_{0}, \tau\right]$ by a constant that does not depend on $\tau$. This implies that $D$ can never reach 0 and, therefore, we can choose $\tau=T$.

Moreover, $C$ satisfies for all the BSDE

$$
\begin{aligned}
-d C_{t}= & -\frac{\mathrm{d} Z_{t}}{\mathrm{~d} x} d W_{t}+f_{x x}\left(t, X_{t}, \alpha_{t}\right) D_{t} d t \\
& +f_{x a}\left(t, X_{t}, \alpha_{t}\right)\left[f_{y x}^{*}\left(t, X_{t}, Y_{t}\right) D_{t}+f_{y y}^{*}\left(t, X_{t}, Y_{t}\right) C_{t}\right] d t,
\end{aligned}
$$

on $\left[t_{0}, T\right]$ with terminal condition $C_{T}=g^{\prime \prime}\left(X_{T}\right) D_{T}$, where $\alpha_{t}:=f_{y}^{*}\left(t, X_{t}, Y_{t}\right), t \in\left[t_{0}, T\right]$. The dynamics of $\Psi$ are now deduced from those of $C$ and $D$ using the product rule. For all 
$t \in\left[t_{0}, T\right]$ it holds

$$
\begin{aligned}
d \Psi_{t}= & D_{t}^{-1} d C_{t}+C_{t} d D_{t}^{-1} \\
= & D_{t}^{-1} \frac{\mathrm{d} Z_{t}}{\mathrm{~d} x} d W_{t}+f_{y y}^{*}\left(t, X_{t}, Y_{t}\right) \Psi_{t}^{2} d t \\
& +\left[f_{y x}^{*}\left(t, X_{t}, Y_{t}\right)-f_{x a}\left(t, X_{t}, \alpha_{t}\right) f_{y y}^{*}\left(t, X_{t}, Y_{t}\right)\right] \Psi_{t} d t \\
& -\left[f_{x x}\left(t, X_{t}, \alpha_{t}\right)+f_{x a}\left(t, X_{t}, \alpha_{t}\right) f_{y x}^{*}\left(t, X_{t}, Y_{t}\right)\right] d t \\
= & D_{t}^{-1} \frac{\mathrm{d} Z_{t}}{\mathrm{~d} x} d W_{t}-f_{x x}\left(t, X_{t}, \alpha_{t}\right) d t \\
& +\left[f_{y y}^{*}\left(t, X_{t}, Y_{t}\right) \Psi_{t}+f_{y x}^{*}\left(t, X_{t}, Y_{t}\right)\right]\left[\Psi_{t}-f_{x a}\left(t, X_{t}, \alpha_{t}\right)\right] d t .
\end{aligned}
$$

Considering (6) in Remark 1.4 we have for all $t \in[0, T], x, y \in[0, \infty)$ that

$$
\begin{aligned}
f_{y y}^{*}(t, x, y) \mathbf{1}_{(0, \infty)}(x) & =\frac{1}{f_{a a}}\left(t, x, f_{y}^{*}(t, x, y)\right) \mathbf{1}_{\left(f_{a}(t, x, 0+), f_{a}\left(t, x, a_{\text {sup }}\right)\right.}(y) \mathbf{1}_{(0, \infty)}(x), \\
f_{y x}^{*}(t, x, y) \mathbf{1}_{(0, \infty)}(x) & =-\frac{f_{a x}}{f_{a a}}\left(t, x, f_{y}^{*}(t, x, y)\right) \mathbf{1}_{\left(f_{a}(t, x, 0+), f_{a}\left(t, x, a_{\mathrm{sup}}\right)\right)}(y) \mathbf{1}_{(0, \infty)}(x) .
\end{aligned}
$$

Together with (20) we obtain for all $t \in\left[t_{0}, T\right]$

$$
d \Psi_{t}=D_{t}^{-1} \frac{\mathrm{d} Z_{t}}{\mathrm{~d} x} d W_{t}-f_{x x}\left(t, X_{t}, \alpha_{t}\right) d t+f_{y y}^{*}\left(t, X_{t}, Y_{t}\right)\left(\Psi_{t}-f_{x a}\left(t, X_{t}, \alpha_{t}\right)\right)^{2} d t .
$$

To sum up, $\Psi$ solves on $\left[t_{0}, T\right]$ the BSDE with terminal condition $\Psi_{T}=g^{\prime \prime}\left(X_{T}\right)$ and driver

$$
\begin{aligned}
h(t, \psi)= & -f_{y y}^{*}\left(t, X_{t}, Y_{t}\right) \psi^{2}+\left[f_{y y}^{*}\left(t, X_{t}, Y_{t}\right) f_{x a}\left(t, X_{t}, \alpha_{t}\right)-f_{y x}^{*}\left(t, X_{t}, Y_{t}\right)\right] \psi \\
& +f_{x x}\left(t, X_{t}, \alpha_{t}\right)+f_{y x}^{*}\left(t, X_{t}, Y_{t}\right) f_{x a}\left(t, X_{t}, \alpha_{t}\right) \\
= & -\frac{\left(\psi-f_{x a}\left(t, X_{t}, \alpha_{t}\right)\right)^{2}}{f_{a a}\left(t, X_{t}, \alpha_{t}\right)} \mathbf{1}_{\left(f_{a}\left(t, X_{t}, 0\right), f_{a}\left(t, X_{t}, a_{\text {sup }}\right)\right)}\left(Y_{t}\right)+f_{x x}\left(t, X_{t}, \alpha_{t}\right) .
\end{aligned}
$$

Lemma 2.11. Let $\mathfrak{L}$ be a common Lipschitz constant for the functions in condition (D1). Then it holds that

$$
0 \leq \Psi_{s} \leq e^{2 \mathfrak{L} T} \mathfrak{L}(T+1)
$$

a.s. for all $s \in\left[t_{0}, T\right]$.

Proof. We first show $\Psi \geq 0$ using the comparison theorem. To this end define

$$
\widehat{h}(t, \psi):=0 \wedge\left(\left(-\frac{\left(\psi-f_{x a}\left(t, X_{t}, \alpha_{t}\right)\right)^{2}}{f_{a a}\left(t, X_{t}, \alpha_{t}\right)}+f_{x x}\left(t, X_{t}, \alpha_{t}\right)\right) \mathbf{1}_{\left(f_{a}\left(t, X_{t}, 0\right), f_{a}\left(t, X_{t}, a_{\text {sup }}\right)\right)}\left(Y_{t}\right)\right)
$$

for all $\psi \in \mathbb{R}$ and $t \in\left[t_{0}, T\right]$. Clearly, it holds that $\hat{h} \leq h$. We claim that the zero process solves the BSDE given by the driver $\widehat{h}$ and the terminal condition 0 . Indeed, plugging $\psi=0$ into the definition of $\widehat{h}$, we notice that for all $t \in\left[t_{0}, T\right]$ it holds

$$
\left(-\frac{f_{x a}^{2}}{f_{a a}}+f_{x x}\right)\left(t, X_{t}, \alpha_{t}\right)=\left(\frac{1}{f_{a a}} \operatorname{det}\left(D^{2} f\right)\right)\left(t, X_{t}, \alpha_{t}\right)
$$

where $D^{2} f$ is the Hessian matrix of $f$ with respect to the two variables $x$ and $a$. $D^{2} f$ is symmetrical and positive semi-definite everywhere, since $f$ is convex. Therefore, the eigenvalues 
of $D^{2} f$ are always non-negative. Moreover, we can calculate $\operatorname{det}\left(D^{2} f\right)$ as the product of those eigenvalues. Therefore, $\hat{h}(t, 0)=0$, showing that 0 is a solution of the BSDE with parameters $(\widehat{h}, 0)$. Finally, the fact that $g_{x x} \geq 0$ and the comparison theorem for BSDEs implies that $\Psi \geq 0$. Indeed, we can use the comparison principle for Lipschitz BSDEs since $\Psi$ is bounded on $\left[t_{0}, T\right]$.

In order to show the upper estimate notice that by Equation (22) for all $\left[t_{0}, T\right]$ and $\psi \in \mathbb{R}$ we have

$$
\begin{aligned}
h(t, \psi) \leq & {\left[f_{y y}^{*}\left(t, X_{t}, Y_{t}\right) f_{x a}\left(t, X_{t}, \alpha_{t}\right)-f_{y x}^{*}\left(t, X_{t}, Y_{t}\right)\right] \psi } \\
& +f_{x x}\left(t, X_{t}, \alpha_{t}\right)+f_{y x}^{*}\left(t, X_{t}, Y_{t}\right) f_{x a}\left(t, X_{t}, \alpha_{t}\right) .
\end{aligned}
$$

Let us remark that

$$
-f_{y y}^{*}(t, \cdot, \cdot) f_{x a}\left(t, \cdot, f_{y}^{*}(t, \cdot, \cdot)\right)+f_{y x}^{*}(t, \cdot, \cdot)
$$

is equal to $\partial_{x} f_{y}^{*}(t, \cdot, \cdot)-\partial_{y}\left(f_{x}\left(t, \cdot, f_{y}^{*}(t, \cdot, \cdot)\right)\right)$ and

$$
f_{x x}\left(t, \cdot, f_{y}^{*}(t, \cdot, \cdot)\right)+f_{y x}^{*}(t, \cdot, \cdot) f_{x a}\left(t, \cdot, f_{y}^{*}(t, \cdot, \cdot)\right)
$$

to $\partial_{x}\left(f_{x}\left(t, \cdot, f_{y}^{*}(t, \cdot, \cdot)\right)\right)$. By condition $(\mathrm{D} 1)$, these functions are bounded by $2 \mathfrak{L}$ and by $\mathfrak{L}$ respectively, and hence $h(t, \psi) \leq 2 \mathcal{L} \psi+\mathcal{L}$. By comparing $\Psi$ with the solution of the BSDE with terminal condition $\mathcal{L}$ and linear driver $2 \mathcal{L} \psi+\mathcal{L}$ we arrive at $(23)$.

According to the assumption (D1) by Lemma 2.11 the process $\Psi$ is non-negative and bounded from above, say by $C \in[0, \infty)$. Notice that $C$ does not depend on $x$ and $t_{0}$. We have $\left|u_{x}\left(t_{0}, x\right)\right|=\left|u_{x}\left(t_{0}, X_{t_{0}}\right)\right|=\left|\Psi_{t_{0}}\right| \leq C$, which is a contradiction to (17) in Theorem 2.8.

Therefore, there exists a unique weakly regular decoupling field $u$ on $[0, T]$ associated with FBSDE (7). According to Theorem 2.6 this implies for all $t \in[0, T]$ and $x \in[0, \infty)$ the existence of a unique solution of (7). The property $u(t, x)=0, x \leq 0$, is a simple consequence of the fact that $(X, Y, Z)=(0,0,0)$ solve the FBSDE with the initial condition $X_{t}=x \leq 0$.

Corollary 2.12. Let $u$ be the unique weakly regular decoupling field associated to (7) from Theorem 2.10. For all $t \in[0, T]$ and $x \in[0, \infty)$ there exists a unique solution $(X, Y, Z)$ of FBSDE (7). The processes $X$ and $Y$ are both bounded and non-negative and hence $(X, Y, Z)$ is a solution to (7) in the sense of Section 1.

Proof. We have seen $X, Y \geq 0$ in the proof of Theorem 2.10. Furthermore, $X$ is decreasing and, therefore, $X \leq x$. Boundedness of $X$ together with the decoupling condition and Lipschitz continuity of $u$ in $x$ as well as $u(\cdot, 0)=0$ imply that $Y$ must also be bounded. Finally, Lipschitz continuity of $f_{y}^{*}$ together with $f_{y}^{*}(\cdot, 0,0)=0$ implies boundedness of $s \mapsto f_{y}^{*}\left(s, X_{s}, Y_{s}\right)$.

\section{Problem (P) in the Lipschitz case}

In this section we turn to problem (P). We solve it by making similar assumptions on $f$ as in Subsection 2.2. More precisely, we assume conditions (C0) - (C4) as well as (D1) and (D2). Moreover, we require the following conditions.

(D3) It holds that $\sup A=\infty$ and $f_{a}(t, x, 0)=0$ for all $(\omega, t, x) \in \Omega \times[0, T] \times[0, \infty)$.

(D4) The whole Hessian matrix $D^{2} f(t, x, a)$ of $f$ w.r.t. $(x, a) \in[0, \infty) \times(0,+\infty)$ is uniformly bounded independently of $(\omega, t, x, a) \in \Omega \times[0, T] \times[0, \infty) \times(0,+\infty)$. 
Note that assumption (D4) is equivalent to assuming that the components $f_{x x}, f_{a a}$ and $f_{x a}=f_{a x}$ of the Hessian $D^{2} f$ are bounded. We denote by $\left\|f_{x x}\right\|_{\infty},\left\|f_{a a}\right\|_{\infty}$ and $\left\|f_{x a}\right\|_{\infty}$ the respective uniform bounds of these mappings.

Due to Remark 1.4 assumption (D3) implies that $f_{y y}^{*}(t, x, y)=\frac{1}{f_{a a}}\left(t, x, f_{y}^{*}(t, x, y)\right)$ for $y>0$, while $f_{y y}^{*}(t, x, y)=0$ if $y \leq 0$. Together with boundedness of $f_{a a}$ this implies that for positive $y$ the value $f_{y y}^{*}(t, x, y)$ can be uniformly bounded away from 0 , while assumption (D1) implies that $f_{y y}^{*}$ is bounded from above by some value $\left\|f_{y y}^{*}\right\|_{\infty} \in[0, \infty)$ as well.

\subsection{Solving problem $(\mathrm{P})$ via a penalization method}

For every $\left(t_{0}, x_{0}\right) \in[0, T) \times(0, \infty)$ and every penalty function $g^{L}(x)=L x^{2}, L>0$, we have, according to Corollary 2.12 (see also Theorem 2.10 and Theorem 2.6) a unique solution $\left(X^{L}, Y^{L}, Z^{L}\right)$ to the FBSDE (7) with initial condition $X_{t_{0}}=x_{0}$, as well as a unique weakly regular decoupling field $u^{L}$ associated with (7). For every $L \in(0, \infty)$ let $v^{L}: \Omega \times[0, T] \times \mathbb{R} \rightarrow \mathbb{R}$ be the value function (4) of problem (3) with penalty function $g^{L}$. According to Proposition 1.6 the strategy $\alpha_{t}^{L}:=f_{y}^{*}\left(t, X_{t}^{L}, Y_{t}^{L}\right), t \in\left[t_{0}, T\right]$, minimizes $J^{L}$ defined in (11), i.e. it holds that

$$
v^{L}\left(t_{0}, x_{0}\right)=J^{L}\left(t_{0}, x_{0}, \alpha^{L}\right)
$$

for non-negative $x_{0}$.

One objective of this section is to show that, under the above assumptions, $\alpha^{L}$ converges for $L \rightarrow \infty$ to an admissible strategy $\alpha^{\infty} \in \mathcal{A}^{0}\left(t_{0}, x_{0}\right)$, which minimizes $\hat{J}\left(t_{0}, x_{0}, \cdot\right)$, i.e. provides an optimal strategy for problem (9). We do so by first proving convergence of $u^{L}$ to some limit $u^{\infty}$ and then showing convergence of $X^{L}$ to a limit $X^{\infty}$. This will finally lead us to the limit $\alpha^{\infty} \in \mathcal{A}^{0}\left(t_{0}, x_{0}\right)$.

Lemma 3.1. There exist constants $C_{1}, C_{2} \in(0, \infty)$, which depend on $T,\left\|f_{y y}^{*}\right\|_{\infty},\left\|f_{x x}\right\|_{\infty}$, $\left\|f_{a a}\right\|_{\infty}$ and $\left\|f_{x a}\right\|_{\infty}$ only and are monotonically increasing in these values, such that for all $L>0, t \in[0, T)$ and a.a. $x>0$ it holds that

$$
\frac{1}{C_{1}\left(\frac{1}{2 L}+(T-t)\right)}=: \kappa_{t}^{L} \leq u_{x}^{L}(t, x) \leq \gamma_{t}:=C_{2}\left(1+\frac{1}{T-t}\right) .
$$

As a consequence, it holds for all $L>0, t \in[0, T)$ and $x>0$ that

$$
x \cdot \kappa_{t}^{L} \leq u^{L}(t, x) \leq x \cdot \gamma_{t} .
$$

Proof. Let $L>0, t \in[0, T)$ and $x>0$ be fixed. Consider the processes $(X, Y, Z)=$ $\left(X^{L}, Y^{L}, Z^{L}\right)$ on $[t, T]$ solving FBSDE (7) with initial condition $X_{t}=x$, terminal condition $g=g^{L}$ and satisfying the decoupling condition via $u=u^{L}$. Define $\Psi_{s}:=u_{x}\left(s, X_{s}\right)$, $s \in[t, T]$. According to the proof of Theorem 2.10 (see (21)) the non-negative process $\Psi$ satisfies for all $s \in[t, T]$

$$
d \Psi_{s}=\tilde{Z}_{s} d W_{s}-\left(f_{x x}\left(s, X_{s}, \alpha_{s}\right)-f_{y y}^{*}\left(s, X_{s}, Y_{s}\right)\left(\Psi_{s}-f_{x a}\left(s, X_{s}, \alpha_{s}\right)\right)^{2}\right) d s
$$

and $\Psi_{T}=g^{\prime \prime}\left(X_{T}\right)=2 L$, where $\alpha_{s}:=f_{y}^{*}\left(s, X_{s}, Y_{s}\right), s \in[t, T]$. Note that $\operatorname{det}\left(D^{2} f\right)=$ $f_{x x} f_{a a}-f_{x a}^{2}$ and $f_{a a}\left(s, X_{s}, \alpha_{s}\right)=\frac{1}{f_{y y}^{*}}\left(s, X_{s}, Y_{s}\right)$ if $Y_{s}>0$. Consequently it holds for all 
$s \in[t, T]$

$$
\begin{aligned}
d \Psi_{s}= & \tilde{Z}_{s} d W_{s}-\left(f_{x x}\left(s, X_{s}, \alpha_{s}\right) \mathbf{1}_{\left\{Y_{s} \leq 0\right\}}\right. \\
& \left.+f_{y y}^{*}\left(s, X_{s}, Y_{s}\right)\left(\operatorname{det}\left(D^{2} f\right)\left(s, X_{s}, \alpha_{s}\right)+2 f_{x a}\left(s, X_{s}, \alpha_{s}\right) \Psi_{s}-\Psi_{s}^{2}\right)\right) d s .
\end{aligned}
$$

We now define a generator $h: \Omega \times[t, T] \times \mathbb{R} \rightarrow \mathbb{R}$ by

$$
h(s, \psi):=f_{x x}\left(s, X_{s}, \alpha_{s}\right) \mathbf{1}_{\left\{Y_{s} \leq 0\right\}}+f_{y y}^{*}\left(s, X_{s}, Y_{s}\right)\left(\operatorname{det}\left(D^{2} f\right)+2 \psi f_{x a}-\psi^{2}\right)\left(s, X_{s}, \alpha_{s}\right),
$$

such that $d \Psi_{s}=\tilde{Z}_{s} d W_{s}-h\left(s, \Psi_{s}\right) d s, s \in[t, T]$. Furthermore, we define a generator $\hat{h}: \mathbb{R} \rightarrow \mathbb{R}$ via

$$
\hat{h}(\psi):=\left\|f_{y y}^{*}\right\|_{\infty}\left(-2\left\|f_{x a}\right\|_{\infty} \psi-\psi^{2}\right) .
$$

Convexity of $f$ ensures that $f_{x x} \geq 0$ and that $\operatorname{det}\left(D^{2} f\right) \geq 0$. Consequently, we obtain that $\hat{h}(\psi) \leq h(s, \psi)$ for all $s \in[t, T]$ and $\psi \in[0, \infty)$. Let $K_{1}:=2\left\|f_{x a}\right\|_{\infty}\left\|f_{y y}^{*}\right\|_{\infty} \in[0, \infty)$. Define the deterministic, bounded and non-negative process $\hat{\psi}:[t, T] \rightarrow[0, \infty)$ via

$$
\hat{\psi}_{s}:=\left(\frac{e^{K_{1}(T-s)}}{2 L}+\left\|f_{y y}^{*}\right\|_{\infty}(T-s) \frac{e^{K_{1}(T-s)}-1}{K_{1}(T-s)}\right)^{-1}, \quad s \in[t, T] .
$$

where an expression of the form $\frac{e^{x}-1}{x}$ is to be replaced by $1=\lim _{x \rightarrow 0} \frac{e^{x}-1}{x}$ in case $x$ is zero. Observe that $\hat{\psi}$ solves the ODE $d \hat{\psi}_{s}=-\hat{h}\left(s, \hat{\psi}_{s}\right) d s, s \in[t, T]$, with terminal condition $\hat{\psi}_{T}=2 L$. The comparison principle implies $\Psi \geq \hat{\psi}$, which in turn implies $\kappa^{L} \leq \Psi$ for an appropriately chosen $C_{1}$ depending on $T K_{1}$ and $\left\|f_{y y}^{*}\right\|_{\infty}$. This proves the lower bound in (25). Together with the fact that $u(\cdot, 0)=0$ this implies directly the lower bound in (26). Furthermore, using Lemma 2.9, we obtain that $Y_{s}=u\left(s, X_{s}\right)>0$ for all $s \in[t, T)$, which simplifies the generator $h$ as the first summand vanishes a.e.

We now prove the upper bound in (25). To this end define another locally Lipschitz generator $\breve{h}: \mathbb{R} \rightarrow \mathbb{R}$ via

$$
\check{h}(\psi):=K_{2}-K_{3} \psi^{2}, \quad \psi \in \mathbb{R},
$$

where $K_{2}:=\left\|f_{x x}\right\|_{\infty}+3\left\|f_{x a}\right\|_{\infty}^{2}\left\|f_{y y}^{*}\right\|_{\infty}+1 \in(0, \infty)$ and $K_{3}:=\frac{1}{2\left\|f_{a a}\right\|_{\infty}} \in(0, \infty)$. It holds that

$$
2 f_{x a} \psi=2\left(\sqrt{2} f_{x a}\right)\left(\frac{1}{\sqrt{2}} \psi\right) \leq 2\left\|f_{x a}\right\|_{\infty}^{2}+\frac{1}{2} \psi^{2} .
$$

This together with the facts that $f_{y y}^{*}(s, x, y) \geq \frac{1}{\left\|f_{a a}\right\|_{\infty}}$ for $y \in(0, \infty)$ and $\operatorname{det}\left(D^{2} f\right)=f_{x x} f_{a a}-$ $f_{x a}^{2}$ implies that $h \leq \check{h}$. Now define the deterministic and bounded process $\check{\psi}:[t, T] \rightarrow \mathbb{R}$ via

$$
\check{\psi}_{s}=\sqrt{\frac{K_{2}}{K_{3}}}\left(1+\frac{2}{\left(1+2\left(2 L \sqrt{\frac{K_{3}}{K_{2}}}-1\right)^{-1}\right) \exp \left(2 \sqrt{K_{2} K_{3}}(T-s)\right)-1}\right), \quad s \in[t, T] .
$$

Then $\check{\psi}$ solves the ODE $d \check{\psi}_{s}=-\check{h}\left(s, \check{\psi}_{s}\right) d s, s \in[t, T]$, with terminal condition $\check{\psi}_{T}=2 L$. The comparison principle implies $\Psi \leq \check{\psi}$. Now observe that $\check{\psi}$ is monotonically increasing in $L$ and converges to

$$
\sqrt{\frac{K_{2}}{K_{3}}}\left(1+\frac{2}{\exp \left(2 \sqrt{K_{2} K_{3}}(T-s)\right)-1}\right)
$$


for all $s \in[t, T]$ as $L \rightarrow \infty$. This implies for all $s \in[t, T]$ that

$$
\Psi_{s} \leq \sqrt{\frac{K_{2}}{K_{3}}}+\frac{1}{K_{3}(T-s)},
$$

which is controlled from above by $\gamma_{s}$ with $C_{2}:=\sqrt{\frac{K_{2}}{K_{3}}} \vee \frac{1}{K_{3}}$. Thus we have proven $\Psi \leq \gamma$ and, therefore, the upper bound in (25). The estimate (26) follows from (25) since $u(t, 0)=0$.

Lemma 3.1 implies in particular that for all $(s, x) \in[0, T) \times(0, \infty)$ the value $\left|u^{L}(s, x)\right|$ can be uniformly bounded independently of $L$ and $\omega$. Also, $u^{L}(s, x)=0$ for $x \leq 0$ according to Theorem 2.10. This together with the next result proves pointwise convergence of $u^{L}$ for $L \rightarrow \infty$.

Lemma 3.2. The mapping $(t, \omega, L, x) \mapsto u^{L}(t, \omega, x)$ is progressively measurable while being continuous and non-decreasing in $L$.

Proof. Consider the FBSDE given by the backward equation as in (7) and the forward equation, which is two-dimensional with the first component being identical to the forward equation in (7) and the second having the form $L_{s}=L_{t}$ for all $s \in[t, T]$, where $L_{t} \in \mathbb{R}$ is the initial value for this second component of the two-dimensional forward equation. Furthermore, instead of $g^{\prime}\left(X_{T}\right)$, we choose the terminal condition to be $\hat{g}(x, L):=(0 \vee(2 x) \wedge C) \cdot(0 \vee L \wedge C), x, L \in \mathbb{R}$, where $C>0$ is an arbitrary but fixed positive constant. Clearly, such an FBSDE satisfies (SLC). We claim that its maximal interval is $[0, T]$. To this end choose arbitrary $t_{0} \in I_{\max }$ and $x=X_{t_{0}}, L=L_{t_{0}} \in \mathbb{R}$ and consider the corresponding $(X, Y, Z)=\left(X^{t_{0}, x, L}, Y^{t_{0}, x, L}, Z^{t_{0}, x, L}\right)$ on $\left[t_{0}, T\right]$. We write

$$
\widehat{C}_{t}=\frac{\mathrm{d} Y_{t}}{\mathrm{~d} L}, \quad \widehat{D}_{t}=\frac{\mathrm{d} X_{t}}{\mathrm{~d} L} \quad \text { and } \quad \gamma_{t}=u_{L}\left(t, X_{t}, L_{t}\right)=\frac{\partial u}{\partial L}\left(t, X_{t}, L_{t}\right), \quad t \in\left[t_{0}, T\right],
$$

where $u: \Omega \times[0, T] \times \mathbb{R} \times \mathbb{R} \rightarrow \mathbb{R}$ is the unique weakly regular decoupling field on $\left[t_{0}, T\right]$. Using the decoupling condition $Y_{t}=u\left(t, X_{t}, L_{t}\right)$ we obtain $\gamma_{t}=\widehat{C}_{t}-\Psi_{t} \widehat{D}_{t}$, where $\Psi_{t}=u_{x}\left(t, X_{t}, L_{t}\right)$, $t \in\left[t_{0}, T\right]$. Observe that $\widehat{D}, \widehat{C}$ and $\Psi$ satisfy similarly to (18) the dynamics

$$
d \widehat{D}_{t}=-\left(f_{y x}^{*}\left(t, X_{t}, Y_{t}\right) \widehat{D}_{t}+f_{y y}^{*}\left(t, X_{t}, Y_{t}\right) \widehat{C}_{t}\right) d t
$$

similarly to (19) the dynamics

$$
\begin{aligned}
-d \widehat{C}_{t}= & -\frac{\mathrm{d} Z_{t}}{\mathrm{~d} L} d W_{t}+f_{x x}\left(t, X_{t}, \alpha_{t}\right) \widehat{D}_{t} d t \\
& +f_{x a}\left(t, X_{t}, \alpha_{t}\right)\left[f_{y x}^{*}\left(t, X_{t}, Y_{t}\right) \widehat{D}_{t}+f_{y y}^{*}\left(t, X_{t}, Y_{t}\right) \widehat{C}_{t}\right] d t
\end{aligned}
$$

and similarly to $(21)$ the dynamics

$$
\begin{aligned}
d \Psi_{t}= & D_{t}^{-1} \frac{\mathrm{d} Z_{t}}{\mathrm{~d} x} d W_{t}-f_{x x}\left(t, X_{t}, \alpha_{t}\right) d t \\
& +f_{y y}^{*}\left(t, X_{t}, Y_{t}\right)\left(\Psi_{t}-f_{x a}\left(t, X_{t}, \alpha_{t}\right)\right)^{2} d t
\end{aligned}
$$


where $\alpha_{t}=f_{y}^{*}\left(t, X_{t}, Y_{t}\right), t \in\left[t_{0}, T\right]$. This implies

$$
\begin{aligned}
d \gamma_{t}= & d \widehat{C}_{t}-\Psi_{t} d \widehat{D}_{t}-\widehat{D}_{t} d \Psi_{t} \\
= & \frac{\mathrm{d} Z_{t}}{\mathrm{~d} L} d W_{t}-f_{x x}\left(t, X_{t}, \alpha_{t}\right) \widehat{D}_{t} d t \\
& -f_{x a}\left(t, X_{t}, \alpha_{t}\right)\left[f_{y x}^{*}\left(t, X_{t}, Y_{t}\right) \widehat{D}_{t}+f_{y y}^{*}\left(t, X_{t}, Y_{t}\right) \widehat{C}_{t}\right] d t \\
& +\Psi_{t}\left(f_{y x}^{*}\left(t, X_{t}, Y_{t}\right) \widehat{D}_{t}+f_{y y}^{*}\left(t, X_{t}, Y_{t}\right) \widehat{C}_{t}\right) d t \\
& -\widehat{D}_{t} D_{t}^{-1} \frac{\mathrm{d} Z_{t}}{\mathrm{~d} x} d W_{t}+\widehat{D}_{t} f_{x x}\left(t, X_{t}, \alpha_{t}\right) d t \\
& -\widehat{D}_{t} f_{y y}^{*}\left(t, X_{t}, Y_{t}\right)\left(\Psi_{t}-f_{x a}\left(t, X_{t}, \alpha_{t}\right)\right)^{2} d t \\
= & f_{y y}^{*}\left(t, X_{t}, Y_{t}\right)\left(\Psi_{t}-f_{x a}\left(t, X_{t}, \alpha_{t}\right)\right) \gamma_{t} d t+\Gamma_{t} d W_{t},
\end{aligned}
$$

where $\Gamma_{t}=\frac{\mathrm{d} Z_{t}}{\mathrm{~d} L}-\widehat{D}_{t} D_{t}^{-1} \frac{\mathrm{d} Z_{t}}{\mathrm{~d} x}, t \in\left[t_{0}, T\right]$. Hence, the process $\gamma$ satisfies a BSDE with linear driver and bounded coefficients as $\Psi$ is uniformly bounded according to the proof of Lemma 2.11. Note that for all $\tilde{x}, \tilde{L} \in \mathbb{R}$ it holds

$$
u(T, \tilde{x}, \tilde{L})=(0 \vee(2 \tilde{x}) \wedge C) \cdot(0 \vee \tilde{L} \wedge C),
$$

such that $u_{x}\left(T, X_{T}, L_{T}\right)$ and $u_{L}\left(T, X_{T}, L_{T}\right)$ are both non-negative and uniformly bounded (with the respective bounds depending on $C$ ). It follows that $u_{L}\left(t_{0}, x, L\right)$ is non-negative and also that $\gamma$ is uniformly bounded, i.e. independently of $t_{0}$.

Therefore, for every $C>0$, we have a unique weakly regular decoupling field $u$ on $[0, T]$ such that $u_{L} \geq 0$. Finally, if we choose the initial values $x, L$ between 0 and $C$ and consider the corresponding FBSDE, we observe that $X_{t}, L_{t}$ both stay in $[0, C]$, such that the terminal condition $Y_{T}=2 X_{T} L$ is satisfied, which means that $X, Y$ solve FBSDE (7) with terminal condition $g^{L}$. Uniqueness of solutions implies $u^{L}\left(t_{0}, x\right)=u\left(t_{0}, x, L\right)$ and the monotonicity of $u$ in $L$ is inherited by $u^{L}$ as $C>0$ can be chosen arbitrarily large.

Now we can define $u^{\infty}: \Omega \times[0, T) \times \mathbb{R} \rightarrow \mathbb{R}$ via

$$
u^{\infty}(s, x):=\lim _{L \rightarrow \infty} u^{L}(s, x), \quad(s, x) \in[0, T) \times \mathbb{R} .
$$

Note that $u^{\infty}$ inherits progressive measurability from $u^{L}$. Also note that for all $s<T$ the mapping $u^{L}(s, \cdot)$ is Lipschitz continuous w.r.t. $x$ with Lipschitz constant $\gamma_{s}$, which does not depend on $L$. Therefore, $u^{\infty}(s, \cdot)$ is also Lipschitz continuous with the same Lipschitz constant. Finally, note that for all $s \in[0, T)$ and all $x \in(0, \infty)$

$$
u^{\infty}(s, x) \geq x \cdot \lim _{L \rightarrow \infty} \kappa_{s}^{L}=\frac{x}{C_{1}(T-s)} .
$$

Now fix an initial condition $\left(t_{0}, x_{0}\right) \in[0, T) \times(0, \infty)$. As above we denote for every $L \in(0, \infty)$ by $\left(X^{L}, Y^{L}, Z^{L}\right)$ the unique solution to the FBSDE (7) with initial condition $X_{t_{0}}=x_{0}$ and penalty function $g^{L}$. In particular, for every $L \in(0, \infty)$ the process $X^{L}$ satisfies by construction the ODE $\dot{X}_{t}^{L}=-f_{y}^{*}\left(t, X_{t}^{L}, u^{L}\left(t, X_{t}^{L}\right)\right), t \in\left[t_{0}, T\right]$. According to Remark 1.3 and Lemma 3.2 the family of functions $f_{y}^{*}\left(t, \cdot, u^{L}(t, \cdot)\right)$ is non-decreasing in $L$. A comparison principle for ODEs then entails that $X^{L}$ is non-increasing in $L$. As $X^{L}$ is non-negative for all 
$L \in(0, \infty)$ by Corollary 2.12 we can define the progressively measurable, non-negative process $X^{\infty}$ as an a.e. limit via

$$
X_{t}^{t_{0}, x_{0}, \infty}:=X_{t}^{\infty}:=\lim _{L \rightarrow \infty} X_{t}^{L}, \quad t \in\left[t_{0}, T\right) .
$$

Note that we refrain from defining $X^{\infty}$ at time $T$ for the time being. This will be done after the next lemma.

Lemma 3.3. It holds that $\lim _{L \rightarrow \infty} u^{L}\left(\cdot, X^{L}\right) \rightarrow u^{\infty}\left(\cdot, X^{\infty}\right)$ almost everywhere on $\Omega \times\left[t_{0}, T\right)$. Moreover, $X^{\infty}$ is the unique solution of the $O D E$

$$
X_{t}^{\infty}=x_{0}-\int_{t_{0}}^{t} f_{y}^{*}\left(s, X_{s}^{\infty}, u^{\infty}\left(s, X_{s}^{\infty}\right)\right) d s, \quad t \in\left[t_{0}, T\right) .
$$

In particular, almost all paths of $X^{\infty}$ are absolutely continuous and non-increasing on $\left[t_{0}, T\right)$.

Proof. First, observe that by Lemma 3.1 it holds for all $s \in\left[t_{0}, T\right)$ that

$$
\begin{gathered}
\left|u^{L}\left(s, X_{s}^{L}\right)-u^{\infty}\left(s, X_{s}^{\infty}\right)\right| \leq\left|u^{L}\left(s, X_{s}^{L}\right)-u^{L}\left(s, X_{s}^{\infty}\right)\right|+\left|u^{L}\left(s, X_{s}^{\infty}\right)-u^{\infty}\left(s, X_{s}^{\infty}\right)\right| \\
\leq \gamma_{s}\left|X_{s}^{L}-X_{s}^{\infty}\right|+\left|u^{L}\left(s, X_{s}^{\infty}\right)-u^{\infty}\left(s, X_{s}^{\infty}\right)\right| .
\end{gathered}
$$

By construction both summands on the right-hand-side converge to 0 as $L \rightarrow \infty$. Dominated convergence implies for all $t \in\left[t_{0}, T\right)$

$$
\lim _{L \rightarrow \infty} \int_{t_{0}}^{t} f_{y}^{*}\left(s, X_{s}^{L}, u^{L}\left(s, X_{s}^{L}\right)\right) d s=\int_{t_{0}}^{t} f_{y}^{*}\left(s, X_{s}^{\infty}, u^{\infty}\left(s, X_{s}^{\infty}\right)\right) d s .
$$

This proves that $X^{\infty}$ satisfies (30). Lipschitz continuity of $f_{y}^{*}$ and of $u^{\infty}$ for compact timeintervals show that $X^{\infty}$ is the unique solution of this ODE. Absolute continuity and monotonicity of the paths of $X^{\infty}$ are direct consequences of (30) and non-negativity of $f_{y}^{*}$.

By Lemma 3.3 the $\operatorname{limit}_{\lim } \rightarrow T X_{t}^{\infty}$ exists and we can continuously extend the process $X^{\infty}$ to the whole of $\left[t_{0}, T\right]$ via $X_{T}^{\infty}:=\lim _{t \rightarrow T} X_{t}^{\infty}$.

Lemma 3.4. It holds that $X_{T}^{\infty}=0$.

Proof. First, note that $X_{T}^{\infty} \geq 0$ a.s. Next, for every fixed $(\omega, s, x)$ the function $f_{y}^{*}(s, x, \cdot)$ : $[0, \infty) \rightarrow[0, \infty)$ starts in zero and is strictly increasing with a derivative which is larger or equal to $\frac{1}{\left\|f_{a a}\right\|_{\infty}}$ almost everywhere. Using (28) and since $X^{\infty}$ has non-increasing paths, we obtain for all $t \in\left[t_{0}, T\right)$

$$
\begin{aligned}
X_{t}^{\infty} & =x_{0}-\int_{t_{0}}^{t} f_{y}^{*}\left(s, X_{s}^{\infty}, u^{\infty}\left(s, X_{s}^{\infty}\right)\right) d s \leq x_{0}-\int_{t_{0}}^{t} \frac{1}{\left\|f_{a a}\right\|_{\infty}} \cdot \frac{X_{s}^{\infty}}{C_{1}(T-s)} d s \\
& \leq x_{0}-\frac{X_{t}^{\infty}}{\left\|f_{a a}\right\|_{\infty} C_{1}} \int_{t_{0}}^{t} \frac{1}{(T-s)} d s .
\end{aligned}
$$

Hence, it holds for $t \in\left[t_{0}, T\right)$

$$
X_{t}^{\infty}\left[1+\frac{1}{\left\|f_{a a}\right\|_{\infty} C_{1}} \int_{t_{0}}^{t} \frac{1}{(T-s)} d s\right] \leq x_{0} .
$$

Taking the limit $t \rightarrow T$ implies that $X_{T}^{\infty}=0$ a.s. 
We next show that the process defined by

$$
\alpha_{s}^{\infty}:=f_{y}^{*}\left(s, X_{s}^{\infty}, u^{\infty}\left(s, X_{s}^{\infty}\right)\right) \text { for } s \in\left[t_{0}, T\right) \text { and } \alpha_{T}^{\infty}:=0
$$

is optimal for problem (P). Notice that Lemma 3.4 implies that $\alpha^{\infty}$ lies in the set $\mathcal{A}^{0}\left(t_{0}, x_{0}\right)$.

Theorem 3.5. Let $\left(t_{0}, x_{0}\right) \in[0, T) \times(0, \infty)$. The strategy $\alpha^{\infty} \in \mathcal{A}^{0}\left(t_{0}, x_{0}\right)$ defined in (31) is an optimal control for problem (9) and satisfies

$$
\hat{v}\left(t_{0}, x_{0}\right)=\hat{J}\left(t_{0}, x_{0}, \alpha^{\infty}\right)<\infty .
$$

Moreover, it holds that $\lim _{L \rightarrow \infty} v^{L}\left(t_{0}, x_{0}\right)=\hat{v}\left(t_{0}, x_{0}\right)$.

Proof. Since the family of functions $g^{L}$ is non-decreasing in $L$ we obtain that also the family $v^{L}$ is non-decreasing in $L$. In particular, the limit $v^{\infty}\left(t_{0}, x_{0}\right):=\lim _{L \rightarrow \infty} v^{L}\left(t_{0}, x_{0}\right)$ exists. Since it holds that

$$
v^{L}\left(t_{0}, x_{0}\right)=\operatorname{essinf}_{\alpha \in \mathcal{A}\left(t_{0}, x_{0}\right)} J^{L}\left(t_{0}, x_{0}, \alpha\right) \leq \operatorname{essinf}_{\alpha \in \mathcal{A}^{0}\left(t_{0}, x_{0}\right)} J^{L}\left(t_{0}, x_{0}, \alpha\right)=\hat{v}\left(t_{0}, x_{0}\right),
$$

it follows that $v^{\infty}\left(t_{0}, x_{0}\right) \leq \hat{v}\left(t_{0}, x_{0}\right)$.

Next, let us as above denote for every $L \in(0, \infty)$ by $\left(X^{L}, Y^{L}, Z^{L}\right)$ the unique solution to the FBSDE (7) with initial condition $X_{t_{0}}=x_{0}$ and penalty function $g^{L}$. According to Proposition 1.6 the strategy $\alpha_{t}^{L}:=f_{y}^{*}\left(t, X_{t}^{L}, Y_{t}^{L}\right), t \in\left[t_{0}, T\right]$, minimizes $J^{L}$ defined in (11). This, together with non-negativity of $g$ implies that

$$
v^{L}\left(t_{0}, x_{0}\right) \geq \mathbb{E}\left[\int_{t_{0}}^{T} f\left(s, X_{s}^{L}, \alpha_{s}^{L}\right) d s \mid \mathcal{F}_{t_{0}}\right] .
$$

Note that $X^{L}$ converges to $X^{\infty}=X^{t_{0}, x_{0}, \alpha^{\infty}}$ a.s. for $L \rightarrow \infty$ by definition. Since $f_{y}^{*}(s, \cdot, \cdot)$ is continuous it follows again from Lemma 3.3 that $\alpha^{L}=f_{y}^{*}\left(\cdot, X^{L}, u^{L}\left(\cdot, X^{L}\right)\right)$ converges for $L \rightarrow \infty$ to $\alpha^{\infty}$ a.e. as well. Due to continuity of $f(s, \cdot, \cdot)$ the process $f\left(\cdot, X_{.}^{L}, \alpha^{L}\right)$ converges to $f\left(\cdot, X^{\infty}, \alpha^{\infty}\right)$ for $L \rightarrow \infty$ almost everywhere. Non-negativity of $f$, Fatou's Lemma and (32) then imply that

$$
v^{\infty}\left(t_{0}, x_{0}\right) \geq \mathbb{E}\left[\int_{t_{0}}^{T} f\left(s, X_{s}^{\infty}, \alpha_{s}^{\infty}\right) d s \mid \mathcal{F}_{t_{0}}\right] \geq \hat{v}\left(t_{0}, x_{0}\right)
$$

This proves that

$$
\hat{v}\left(t_{0}, x_{0}\right)=v^{\infty}\left(t_{0}, x_{0}\right)=\mathbb{E}\left[\int_{t_{0}}^{T} f\left(s, X_{s}^{\infty}, \alpha_{s}^{\infty}\right) d s \mid \mathcal{F}_{t_{0}}\right]
$$

Finally, observe that finiteness of $\hat{v}\left(t_{0}, x_{0}\right)$ is a consequence of assumption (C3). Indeed, taking the control $\alpha_{s}=\frac{x_{0}}{T-t_{0}}, s \in\left[t_{0}, T\right]$, shows that

$$
\hat{v}\left(t_{0}, x_{0}\right) \leq \mathbb{E}\left[\int_{t_{0}}^{T} f\left(s, \frac{T-s}{T-t_{0}} x_{0}, \frac{x_{0}}{T-t_{0}}\right) d s \mid \mathcal{F}_{t_{0}}\right]<\infty .
$$

We finally show that the limit decoupling field $u^{\infty}$ is the weak derivative of the value function $\hat{v}$ with respect to the $x$-variable. 
Theorem 3.6. The value function for Problem $(P)$ satisfies for almost all $(t, x)$

$$
\hat{v}(t, x)=\int_{0}^{x} u^{\infty}(t, z) \mathrm{d} z, \quad \text { a.s. }
$$

Proof. We first prove that for all $L>0$ the value function $v^{L}$ is weakly differentiable in $x$ with the weak derivative $u^{L}$. To this end recall that $\alpha_{s}^{L}=f_{y}^{*}\left(s, X_{s}^{L}, Y_{s}^{L}\right)$ is optimal in (3) with penalty function $g^{L}$ and hence

$$
v^{L}(t, x)=\mathbb{E}\left[\int_{t}^{T} f\left(s, X_{s}^{L}, \alpha_{s}^{L}\right) d s+L\left(X_{T}^{L}\right)^{2} \mid \mathcal{F}_{t}\right] .
$$

The strong regularity of $u^{L}$ entails that $X^{L}$ and $Y^{L}$ are weakly differentiable w.r.t. $x$. It is now straightforward to show that $v^{L}$ is weakly differentiable and that we can differentiate under the integral in (34) (see e.g. Lemma A.2.5. and Lemma A.2.4. of [10]). The weak derivative of $v^{L}$ with respect to $x$ is thus given by

$$
v_{x}^{L}(t, x)=\mathbb{E}\left[\int_{t}^{T}\left(f_{x}\left(s, X_{s}^{L}, \alpha_{s}^{L}\right) \frac{\mathrm{d}}{\mathrm{d} x} X_{s}^{L}-f_{a}\left(s, X_{s}^{L}, \alpha_{s}^{L}\right) \frac{\mathrm{d}}{\mathrm{d} x} \alpha_{s}^{L}\right) d s-2 L X_{T}^{L} \frac{\mathrm{d}}{\mathrm{d} x} X_{T}^{L} \mid \mathcal{F}_{t}\right] .
$$

Notice that $f_{a}\left(s, X_{s}^{L}, \alpha_{s}^{L}\right)=f_{a}\left(s, X_{s}^{L}, f_{a}^{-1}\left(s, X_{s}^{L}, Y_{s}^{L}\right)\right)=Y_{s}^{L}$. Also observe that $2 L X_{T}^{L}=$ $Y_{T}^{L}$. By applying the product rule to $Y^{L} \frac{\mathrm{d}}{\mathrm{d} x} X^{L}$ on the right-hand side of equation (35), we further obtain $v_{x}^{L}(t, x)=Y_{t}^{L}=u^{L}(t, x)$. With the fundamental theorem of calculus for weak derivatives (see e.g. Lemma A.2.1. of [10]) we get $v^{L}(t, x)=\int_{0}^{x} u^{L}(t, z) \mathrm{d} z$ a.e. Since $u^{L}$ converges from below to $u^{\infty}$, monotone convergence implies equation (33).

Example 3.7. Let $C \in(1, \infty)$ and let $\eta, \gamma:[0, T] \times \Omega \rightarrow[0, \infty)$ be progressively measurable stochastic processes such that for all $t \in[0, T]$ it holds a.s. that $\eta_{t} \geq \frac{1}{C}$ and $\max \left(\eta_{t}, \gamma_{t}\right) \leq C$. Assume that for all $t \in[0, T], x \in \mathbb{R}$ and $a \in \mathbb{R}$ it holds that $f(t, x, a)=\eta_{t} \frac{|a|^{3}+2|a|^{2}}{|a|+1}+\gamma_{t}|x|^{2}$. Then conditions (C0), (C1), (C2), (C3) and (C4) are satisfied. Moreover, it holds for all $t \in[0, T], x \in \mathbb{R}$ and $a \in \mathbb{R}$ a.s. that $\frac{2}{C} \leq 2 \eta_{t} \leq f_{a a}(t, x, a) \leq 4 \eta_{t} \leq 4 C$. This together with (6) implies that $[0, \infty)^{2} \ni(x, y) \mapsto f_{y}^{*}(t, x, y)$ is uniformly Lipschitz continuous. It follows that (D1) is satisfied. Moreover, it follows that (D4) is satisfied. Observe that $f_{a}(t, x, a)=0$ if and only if $a=0$. This implies (D2). Condition (D3) is also satisfied. Therefore, it follows from Theorem 3.5 that $\alpha^{\infty}$ is an optimal control in Problem (P).

\subsection{The FBSDE associated to Problem (P)}

We have shown in Subsection 3.1 that for every $\left(t_{0}, x_{0}\right) \in[0, T) \times(0, \infty)$ the solution component $X^{L}$ of the FBSDE (7) with initial condition $X_{t_{0}}^{L}=x_{0}$ and penalty function $g^{L}$ converges to $X^{\infty}$ as $L \rightarrow \infty$. Moreover, the associated decoupling field $u^{L}$ converges to $u^{\infty}$. This allows to define a process $Y^{\infty}$ via $Y^{\infty}=u^{\infty}\left(\cdot, X^{\infty}\right)$. The next result shows that also the solution component $Z^{L}$ has a limit $Z^{\infty}$ and that the process $\left(X^{\infty}, Y^{\infty}, Z^{\infty}\right)$ satisfies a system of coupled SDEs. In contrast to (7), there are two constraints imposed on the forward process $X^{\infty}$, while $Y^{\infty}$ is not required to satisfy any boundary conditions.

Theorem 3.8. Let $\left(t_{0}, x_{0}\right) \in[0, T) \times(0, \infty)$. For every $L \in(0, \infty)$ let $\left(X^{L}, Y^{L}, Z^{L}\right)$ be the solution of the FBSDE (7) with initial condition $X_{t_{0}}^{L}=x_{0}$ and penalty function $g^{L}$ and 
let $u^{L}$ be the associated decoupling field. Let $u^{\infty}$ and $X^{\infty}$ be the limits of $u^{L}$ and $X^{L}$ as $L \rightarrow \infty$ defined in (27) and (29). Let $Y^{\infty}: \Omega \times[0, T) \rightarrow \mathbb{R}$ satisfy for all $t \in[0, T)$ a.s. that $Y_{t}^{\infty}=u^{\infty}\left(t, X_{t}^{\infty}\right)$. Then the sequence $Z^{L}$ converges in $L^{2}\left(\left(t_{0}, t\right) \times \Omega, \mathbb{R}^{d}\right)$ for any $t \in\left[t_{0}, T\right)$ to $Z^{\infty}$ and the process $\left(X^{\infty}, Y^{\infty}, Z^{\infty}\right)$ satisfies for all $t_{0} \leq t \leq r<T$ a.s. that

$$
\begin{aligned}
X_{t}^{\infty} & =x_{0}-\int_{t_{0}}^{t} f_{y}^{*}\left(s, X_{s}^{\infty}, Y_{s}^{\infty}\right) d s, \quad X_{T}^{\infty}=\lim _{s \rightarrow T} X_{s}^{\infty}=0 \\
Y_{t}^{\infty} & =Y_{r}^{\infty}+\int_{t}^{r} f_{x}\left(s, X_{s}^{\infty}, f_{y}^{*}\left(s, X_{s}^{\infty}, Y_{s}^{\infty}\right)\right) d s-\int_{t}^{r} Z_{s}^{\infty} d W_{s} .
\end{aligned}
$$

Proof. Let $t_{0} \leq t \leq r<T$. The fact that $X^{\infty}$ satisfies $X_{t}^{\infty}=x_{0}-\int_{t_{0}}^{t} f_{y}^{*}\left(s, X_{s}^{\infty}, Y_{s}^{\infty}\right) d s$ follows from Lemma 3.3 and the definition of $Y^{\infty}$. By Lemma 3.4 it holds that $X_{T}^{\infty}=0$. It follows from Theorem 2.10 that for all $L>0$ it holds

$$
Y_{t}^{L}=Y_{r}^{L}+\int_{t}^{r} f_{x}\left(s, X_{s}^{L}, f_{y}^{*}\left(s, X_{s}^{L}, Y_{s}^{L}\right)\right) d s-\int_{t}^{r} Z_{s}^{L} d W_{s} .
$$

Applying Itô's formula for $L \leq L^{\prime}$ we have

$$
\begin{aligned}
\left|Y_{t}^{L}-Y_{t}^{L^{\prime}}\right|^{2} & =\left|Y_{r}^{L}-Y_{r}^{L^{\prime}}\right|^{2}-\int_{t}^{r}\left|Z_{s}^{L}-Z_{s}^{L^{\prime}}\right|^{2} d s-\int_{t}^{r}\left(Z_{s}^{L}-Z_{s}^{L^{\prime}}\right) d W_{s} \\
& +2 \int_{t}^{r}\left(Y_{s}^{L}-Y_{s}^{L^{\prime}}\right)\left(f_{x}\left(s, X_{s}^{L}, f_{y}^{*}\left(s, X_{s}^{L}, Y_{s}^{L}\right)\right)-f_{x}\left(s, X_{s}^{L^{\prime}}, f_{y}^{*}\left(s, X_{s}^{L^{\prime}}, Y_{s}^{L^{\prime}}\right)\right)\right) d s .
\end{aligned}
$$

By assumption (D1) there exists $C>0$ such that for all $s \in[t, r]$ and $0<L \leq L^{\prime}$

$$
\left|f_{x}\left(s, X_{s}^{L}, f_{y}^{*}\left(s, X_{s}^{L}, Y_{s}^{L}\right)\right)-f_{x}\left(s, X_{s}^{L^{\prime}}, f_{y}^{*}\left(s, X_{s}^{L^{\prime}}, Y_{s}^{L^{\prime}}\right)\right)\right| \leq C\left(\left|X_{s}^{L}-X_{s}^{L^{\prime}}\right|+\left|Y_{s}^{L}-Y_{s}^{L^{\prime}}\right|\right) .
$$

Thereby with Young's inequality and taking the expectation, we obtain

$$
\begin{aligned}
\mathbb{E} \int_{t}^{r}\left|Z_{s}^{L}-Z_{s}^{L^{\prime}}\right|^{2} d s \leq & \mathbb{E}\left|Y_{r}^{L}-Y_{r}^{L^{\prime}}\right|^{2} \\
& \quad+2 C \mathbb{E} \int_{t}^{r}\left|Y_{s}^{L}-Y_{s}^{L^{\prime}}\right|\left(\left|X_{s}^{L}-X_{s}^{L^{\prime}}\right|+\left|Y_{s}^{L}-Y_{s}^{L^{\prime}}\right|\right) d s \\
\leq & \mathbb{E}\left|Y_{r}^{L}-Y_{r}^{L^{\prime}}\right|^{2} \\
& \quad+\left(2 C+C^{2}\right) \mathbb{E} \int_{t}^{r}\left|Y_{s}^{L}-Y_{s}^{L^{\prime}}\right|^{2} d s+\mathbb{E} \int_{t}^{r}\left|X_{s}^{L}-X_{s}^{L^{\prime}}\right|^{2} d s .
\end{aligned}
$$

Lemma 3.3 implies that $X_{t}^{L} \rightarrow X_{t}^{\infty}$ and $Y_{t}^{L} \rightarrow Y_{t}^{\infty}$ for $L \rightarrow \infty$ a.s. Moreover, it holds for all $s \in[t, r]$ and $L>0$ that $\max \left(X_{s}^{L}, X_{s}^{\infty}\right) \leq x_{0}$ and by (26) that $\max \left(\left|Y_{s}^{L}\right|,\left|Y_{s}^{\infty}\right|\right) \leq \gamma_{r} x_{0}$. By the dominated convergence theorem, the two sequences $X^{L}$ and $Y^{L}$ also converge in the space $L^{2}\left(\left(t_{0}, r\right) \times \Omega, \mathbb{R}^{d}\right)$. Combining this with (38), we deduce that $Z^{L}$ is a Cauchy sequence in $L^{2}\left(\left(t_{0}, r\right) \times \Omega, \mathbb{R}^{d}\right)$ and thus converges to $Z^{\infty}$. It is straightforward that the limit $Z^{\infty}$ does not depend on the particular choice of $r<T$.

Again by assumption (D1)

$$
\left|f_{x}\left(s, X_{s}^{L}, f_{y}^{*}\left(s, X_{s}^{L}, Y_{s}^{L}\right)\right)-f_{x}\left(s, X_{s}^{\infty}, f_{y}^{*}\left(s, X_{s}^{\infty}, Y_{s}^{\infty}\right)\right)\right| \leq C\left(\left|X_{s}^{L}-X_{s}^{\infty}\right|+\left|Y_{s}^{L}-Y_{s}^{\infty}\right|\right),
$$

and we can take the limit $L \rightarrow \infty$ in (37) to obtain

$$
Y_{t}^{\infty}=Y_{r}^{\infty}+\int_{t}^{r} f_{x}\left(s, X_{s}^{\infty}, f_{y}^{*}\left(s, X_{s}^{\infty}, Y_{s}^{\infty}\right)\right) d s-\int_{t}^{r} Z_{s}^{\infty} d W_{s} .
$$

This completes the proof. 
Remark 3.9. It follows from (36), that the process

$$
Y^{\infty}+\int_{t_{0}}^{\cdot} f_{x}\left(s, X_{s}^{\infty}, f_{y}^{*}\left(s, X_{s}^{\infty}, Y_{s}^{\infty}\right)\right) d s
$$

is a non-negative local martingale on $\left[t_{0}, T\right)$. Hence its limit at time $T$ exists in $[0, \infty)$ a.s. By the monotone convergence theorem, the integral has also a limit. Since both terms are non-negative, we deduce that the $\operatorname{limit} Y_{T}^{\infty}:=\lim _{t \rightarrow T} Y_{t} \in[0, \infty)$ exists a.s. Note, however, that $Y_{T}^{\infty}$ is not a given terminal condition, but part of the solution.

Remark 3.10. With Lemma 3.1 and Remark 3.9 we can describe the asymptotic behavior of $X_{t}^{\infty}$ as $t \rightarrow T$. Indeed, it follows from the fact that $Y_{t}^{\infty}=u^{\infty}\left(t, X_{t}^{\infty}\right)$ for all $t \in\left[t_{0}, T\right)$ and from (26) that there exist $C_{1}, C_{2} \in(0, \infty)$ such that for all $t \in[0, T)$ it holds

$$
C_{1} \frac{X_{t}^{\infty}}{T-t} \leq u^{\infty}\left(t, X_{t}^{\infty}\right)=Y_{t}^{\infty} \leq X_{t}^{\infty} \cdot C_{2}\left(1+\frac{1}{T-t}\right) .
$$

In particular, it holds that

$$
\frac{1}{C_{2}} Y_{T}^{\infty} \leq \liminf _{t \rightarrow T} \frac{X_{t}^{\infty}}{T-t} \leq \limsup _{t \rightarrow T} \frac{X_{t}^{\infty}}{T-t} \leq \frac{1}{C_{1}} Y_{T}^{\infty} .
$$

Moreover, it follows that

$$
\liminf _{t \rightarrow T}\left(\frac{Y_{t}^{\infty}}{X_{t}^{\infty}}\right)=+\infty .
$$

Note that this behavior is similar to the weak terminal condition (1.3) in [2].

Next, we characterize $\left(X^{\infty}, Y^{\infty}, X^{\infty}\right)$ as the unique solution of (36) within a class of processes satisfying suitable integrability assumptions.

Theorem 3.11. Let $\left(t_{0}, x_{0}\right) \in[0, T) \times(0, \infty)$. The process $\left(X^{\infty}, Y^{\infty}, Z^{\infty}\right)$ constructed in Theorem 3.8 is the unique solution of (36) satisfying $X^{\infty}, Y^{\infty} \geq 0, Z^{\infty} \in L^{2}\left(\left(t_{0}, t\right) \times \Omega, \mathbb{R}^{d}\right)$ for all $t \in\left[t_{0}, T\right)$ and $f_{y}^{*}\left(\cdot, X^{\infty}, Y^{\infty}\right) \in L^{2}\left(\left(t_{0}, T\right) \times \Omega, \mathbb{R}\right)$.

Proof. We first show that $\alpha^{\infty} \in L^{2}\left(\left(t_{0}, T\right) \times \Omega, \mathbb{R}\right)$, where $\alpha^{\infty}$ is defined as in equation (31). First, note that the Lipschitz continuity of $f_{y}^{*}(t, x, \cdot)$ (cf. assumption (D1)), assumptions (D3) and (D4) and (6) prove that there exists a constant $\lambda \in(0, \infty)$ such that for all $t \in[0, T]$, $x, y \in[0, \infty)$ it holds a.s.

$$
f_{a a}\left(t, x, f_{y}^{*}(t, x, y)\right) \geq \lambda .
$$

This, together with assumptions (C1) and (D3), shows that for all $t \in[0, T], x, a \in[0, \infty)$ it holds a.s.

$$
f(t, x, a)=f(t, x, 0)+f_{a}(t, x, 0) a+\int_{0}^{a}(a-s) f_{a a}(t, x, s) d s \geq \frac{\lambda}{2} a^{2},
$$

It now follows from Theorem 3.5 that $\alpha^{\infty}$ is square-integrable.

We now turn to the uniqueness proof. To this end let $(\widehat{X}, \widehat{Y}, \widehat{Z})$ be another solution of (36) satisfying $\widehat{X}, \widehat{Y} \geq 0, \widehat{Z} \in L^{2}\left(\left(t_{0}, t\right) \times \Omega, \mathbb{R}^{d}\right)$ for all $t \in\left[t_{0}, T\right)$ and $f_{y}^{*}(\cdot, \widehat{X}, \widehat{Y}) \in$ $L^{2}\left(\left(t_{0}, T\right) \times \Omega, \mathbb{R}\right)$. Let us denote by $\widehat{\alpha}$ the process satisfying $\widehat{\alpha}_{s}=f_{y}^{*}\left(s, \widehat{X}_{s}, \widehat{Y}_{s}\right), s \in\left[t_{0}, T\right]$. 
Our objective is to prove that $(\widehat{X}, \widehat{Y}, \widehat{Z})$ coincides with $\left(X^{\infty}, Y^{\infty}, X^{\infty}\right)$ by using Gronwall's lemma. To do so, we first show

$$
\lim _{t \rightarrow T} \mathbb{E}\left[\left(Y_{t}^{\infty}-\widehat{Y}_{t}\right)\left(X_{t}^{\infty}-\widehat{X}_{t}\right)\right]=0 .
$$

Recall that for every fixed $(\omega, s, x)$ the function $f_{y}^{*}(s, x, \cdot):[0, \infty) \rightarrow[0, \infty)$ starts in zero and is strictly increasing with a derivative which is larger or equal to $\frac{1}{\left\|f_{a a}\right\|_{\infty}}$ almost everywhere. This implies for all $t \in\left[t_{0}, T\right), r \in[t, T)$ a.s.

$$
\widehat{X}_{t} \geq \widehat{X}_{t}-\widehat{X}_{r}=\int_{t}^{r} f_{y}^{*}\left(s, \widehat{X}_{s}, \widehat{Y}_{s}\right) d s \geq \frac{1}{\left\|f_{a a}\right\|_{\infty}} \int_{t}^{r} \widehat{Y}_{s} d s .
$$

Consequently, with the product rule we obtain for all $t \in\left[t_{0}, T\right), r \in[t, T)$ a.s.

$$
\begin{aligned}
(T-t) \widehat{Y}_{t} & =(T-r) \widehat{Y}_{r}+\int_{t}^{r} \widehat{Y}_{s} d s+\int_{t}^{r}(T-s) f_{x}\left(s, \widehat{X}_{s}, \widehat{\alpha}_{s}\right) d s-\int_{t}^{r}(T-s) \widehat{Z}_{s} d W_{s} \\
& \leq(T-r) \widehat{Y}_{r}+\left\|f_{a a}\right\|_{\infty} \widehat{X}_{t}+\int_{t}^{r}(T-s) f_{x}\left(s, \widehat{X}_{s}, \widehat{\alpha}_{s}\right) d s-\int_{t}^{r}(T-s) \widehat{Z}_{s} d W_{s}
\end{aligned}
$$

Taking conditional expectations we obtain for all $t \in\left[t_{0}, T\right), r \in[t, T)$

$$
(T-t) \widehat{Y}_{t} \leq \mathbb{E}\left[(T-r) \widehat{Y}_{r} \mid \mathcal{F}_{t}\right]+\left\|f_{a a}\right\|_{\infty} \widehat{X}_{t}+\mathbb{E}\left[\int_{t}^{r}(T-s) f_{x}\left(s, \widehat{X}_{s}, \widehat{\alpha}_{s}\right) d s \mid \mathcal{F}_{t}\right]
$$

Notice that the last conditional expectation is well defined as the process $f_{x}(\cdot, \widehat{X}, \widehat{\alpha})$ is nonnegative by $(\mathrm{C} 1)$ and the non-negativity of $\widehat{X}, \widehat{Y}$.

The non-negativity of $f_{x}(\cdot, \widehat{X}, \widehat{\alpha})$ further entails that $\widehat{Y}$ is a non-negative supermartingale on $\left[t_{0}, T\right)$ and hence $0 \leq \mathbb{E}\left[\widehat{Y}_{r} \mid \mathcal{F}_{t}\right] \leq \widehat{Y}_{t}$ a.s. for all $t \in\left[t_{0}, T\right)$ and $r \in[t, T)$. Consequently, $\lim _{r \rightarrow T} \mathbb{E}\left[(T-r) \widehat{Y}_{r} \mid \mathcal{F}_{t}\right]=0$. Thus, by letting $r \uparrow T$ in (41), we obtain for all $t \in\left[t_{0}, T\right)$

$$
\begin{aligned}
(T-t) \widehat{Y}_{t} & \leq\left\|f_{a a}\right\|_{\infty} \widehat{X}_{t}+\mathbb{E}\left[\int_{t}^{T}(T-s) f_{x}\left(s, \widehat{X}_{s}, \widehat{\alpha}_{s}\right) d s \mid \mathcal{F}_{t}\right] \\
& \leq\left\|f_{a a}\right\|_{\infty} \widehat{X}_{t}+(T-t) \mathbb{E}\left[\int_{t}^{T} f_{x}\left(s, \widehat{X}_{s}, \widehat{\alpha}_{s}\right) d s \mid \mathcal{F}_{t}\right] .
\end{aligned}
$$

Observe that this inequality also holds true with $(\widehat{X}, \widehat{Y}, \widehat{\alpha})$ replaced by $\left(X^{\infty}, Y^{\infty}, \alpha^{\infty}\right)$.

It follows from assumptions (D2) and (D4) that for all $t \in\left[t_{0}, T\right], x \in\left[0, x_{0}\right]$ and $a \in[0, \infty)$ it holds

$$
\left|f_{x}(t, x, a)\right| \leq\left\|f_{x x}\right\|_{\infty} x_{0}+\left\|f_{x a}\right\|_{\infty} a .
$$

This together with the assumption that $\hat{\alpha} \in L^{2}\left(\left(t_{0}, T\right) \times \Omega, \mathbb{R}\right)$ ensures that

$$
\mathbb{E}\left[\int_{t_{0}}^{T} f_{x}\left(s, \widehat{X}_{s}, \widehat{\alpha}_{s}\right) d s\right]<\infty .
$$

Next, observe that Hölder's inequality and Jensen's inequality prove for all $t \in\left[t_{0}, T\right)$ that

$$
\mathbb{E}\left[X_{t}^{\infty} \widehat{X}_{t}\right] \leq \sqrt{\mathbb{E}\left[\left(X_{t}^{\infty}\right)^{2}\right] \mathbb{E}\left[\widehat{X}_{t}^{2}\right]} \leq(T-t) \sqrt{\mathbb{E}\left[\int_{t}^{T}\left(\alpha_{r}^{\infty}\right)^{2} d r\right] \mathbb{E}\left[\int_{t}^{T}\left(\widehat{\alpha}_{r}\right)^{2} d r\right]}
$$


This, together with $\widehat{\alpha}, \alpha^{\infty} \in L^{2}\left(\left(t_{0}, T\right) \times \Omega, \mathbb{R}\right)$, implies

$$
\lim _{t \rightarrow T} \frac{1}{T-t} \mathbb{E}\left[X_{t}^{\infty} \widehat{X}_{t}\right]=0
$$

Equation (42) yields

$$
\begin{aligned}
\mathbb{E}\left[\widehat{Y}_{t} X_{t}^{\infty}\right] & \leq \mathbb{E}\left[\left\|f_{a a}\right\|_{\infty} \frac{\widehat{X}_{t} X_{t}^{\infty}}{T-t}+\widehat{X}_{t} \mathbb{E}\left[\int_{t}^{T} f_{x}\left(s, \widehat{X}_{s}, \widehat{\alpha}_{s}\right) d s \mid \mathcal{F}_{t}\right]\right] \\
& \leq\left\|f_{a a}\right\|_{\infty} \mathbb{E}\left[\frac{\widehat{X}_{t} X_{t}^{\infty}}{T-t}\right]+x_{0} \mathbb{E}\left[\int_{t}^{T} f_{x}\left(s, \widehat{X}_{s}, \widehat{\alpha}_{s}\right) d s\right] .
\end{aligned}
$$

With (44) and (43) we obtain $\lim _{t \uparrow T} \mathbb{E}\left[\widehat{Y}_{t} X_{t}^{\infty}\right]=0$.

Similarly, one can show $\lim _{t \uparrow T} \mathbb{E}\left[\widehat{Y}_{t} \widehat{X}_{t}\right]=\lim _{t \uparrow T} \mathbb{E}\left[Y_{t}^{\infty} X_{t}^{\infty}\right]=\lim _{t \uparrow T} \mathbb{E}\left[Y_{t}^{\infty} \widehat{X}_{t}\right]=0$, which entails (40).

Finally, we show that there exists a constant $C \in[0, \infty)$ such that

$$
\left|\alpha_{s}^{\infty}-\widehat{\alpha}_{s}\right| \leq C\left|X_{s}^{\infty}-\widehat{X}_{s}\right|, \quad s \in\left[t_{0}, T\right],
$$

almost everywhere. It then follows from Gronwall's lemma that $\alpha^{\infty}=\widehat{\alpha}$ and $X^{\infty}=\widehat{X}$ and hence also $Y^{\infty}=\widehat{Y}$ and $Z^{\infty}=\widehat{Z}$.

To prove (45) observe that the product rule implies

$$
\begin{aligned}
& \mathbb{E}\left[\left(Y_{t}^{\infty}-\widehat{Y}_{t}\right)\left(X_{t}^{\infty}-\widehat{X}_{t}\right)\right] \\
& \quad=-\mathbb{E}\left[\int_{t_{0}}^{t}\left(f_{x}\left(s, X_{s}^{\infty}, \alpha_{s}^{\infty}\right)-f_{x}\left(s, \widehat{X}_{s}, \widehat{\alpha}_{s}\right)\right)\left(X_{s}^{\infty}-\widehat{X}_{s}\right)+\left(Y_{s}^{\infty}-\widehat{Y}_{s}\right)\left(\alpha_{s}^{\infty}-\widehat{\alpha}_{s}\right) \mathrm{d} s\right],
\end{aligned}
$$

where the expectation of the Brownian integral part vanishes since $Z^{\infty}, \widehat{Z} \in L^{2}\left(\left(t_{0}, t\right) \times \Omega, \mathbb{R}^{d}\right)$ and $X^{\infty}$ and $\widehat{X}$ are bounded. Notice that $Y_{s}^{\infty}=f_{a}\left(s, X_{s}^{\infty}, \alpha_{s}^{\infty}\right)$ and $\widehat{Y}_{s}=f_{a}\left(s, \widehat{X}, \widehat{\alpha}_{s}\right)$. Furthermore, convexity of $f$ ensures that for all $t \in[0, T]$ and $a, \widehat{a}, x, \widehat{x} \in[0, \infty)$ we have

$$
\left(f_{x}(t, x, a)-f_{x}(t, \widehat{x}, \widehat{a})\right)(x-\widehat{x})+\left(f_{a}(t, x, a)-f_{a}(t, \widehat{x}, \widehat{a})\right)(a-\widehat{a}) \geq 0 .
$$

Monotone convergence and (40) yield, by letting $t \uparrow T$ in (46),

$$
\mathbb{E}\left[\int_{t_{0}}^{T}\left(f_{x}\left(s, X_{s}^{\infty}, \alpha_{s}^{\infty}\right)-f_{x}\left(s, \widehat{X}_{s}, \widehat{\alpha}_{s}\right)\right)\left(X_{s}^{\infty}-\widehat{X}_{s}\right)+\left(Y_{s}^{\infty}-\widehat{Y}_{s}\right)\left(\alpha_{s}^{\infty}-\widehat{\alpha}_{s}\right) \mathrm{d} s\right]=0,
$$

which further implies

$$
\left(f_{x}\left(s, X_{s}^{\infty}, \alpha_{s}^{\infty}\right)-f_{x}\left(s, \widehat{X}_{s}, \widehat{\alpha}_{s}\right)\right)\left(X_{s}^{\infty}-\widehat{X}_{s}\right)+\left(f_{a}\left(s, X_{s}^{\infty}, \alpha_{s}^{\infty}\right)-f_{a}\left(s, \widehat{X}, \widehat{\alpha}_{s}\right)\right)\left(\alpha_{s}^{\infty}-\widehat{\alpha}_{s}\right)=0,
$$

almost everywhere. Using strict monotonicity of $f_{a}$ and Lipschitz continuity of $f_{x}$ and $f_{a}$ (with Lipschitz constant $\mathfrak{L}$ ) we obtain

$$
\begin{aligned}
& \lambda\left|\alpha_{s}^{\infty}-\widehat{\alpha}_{s}\right|^{2} \\
\leq & \left(f_{a}\left(s, X_{s}^{\infty}, \alpha_{s}^{\infty}\right)-f_{a}\left(s, X_{s}^{\infty}, \widehat{\alpha}_{s}\right)\right)\left(\alpha_{s}^{\infty}-\widehat{\alpha}_{s}\right) \\
= & \left(f_{a}\left(s, X_{s}^{\infty}, \alpha_{s}^{\infty}\right)-f_{a}\left(s, \widehat{X}_{s}, \widehat{\alpha}_{s}\right)\right)\left(\alpha_{s}^{\infty}-\widehat{\alpha}_{s}\right)+\left(f_{a}\left(s, X_{s}^{\infty}, \widehat{\alpha}_{s}\right)-f_{a}\left(s, \widehat{X}, \widehat{\alpha}_{s}\right)\right)\left(\alpha_{s}^{\infty}-\widehat{\alpha}_{s}\right) \\
= & -\left(f_{x}\left(s, X_{s}^{\infty}, \alpha_{s}^{\infty}\right)-f_{x}\left(s, \widehat{X}_{s}, \widehat{\alpha}_{s}\right)\right)\left(X_{s}^{\infty}-\widehat{X}_{s}\right)+\left(f_{a}\left(s, X_{s}^{\infty}, \widehat{\alpha}_{s}\right)-f_{a}\left(s, \widehat{X}, \widehat{\alpha}_{s}\right)\right)\left(\alpha_{s}^{\infty}-\widehat{\alpha}_{s}\right) \\
\leq & \mathfrak{L}\left(\left|X_{s}^{\infty}-\widehat{X}_{s}\right|+\left|\alpha_{s}^{\infty}-\widehat{\alpha}_{s}\right|\right)\left|X_{s}^{\infty}-\widehat{X}_{s}\right|+\mathfrak{L}\left|X_{s}^{\infty}-\widehat{X}_{s}\right|\left|\alpha_{s}^{\infty}-\widehat{\alpha}_{s}\right| \\
\leq & \left(\mathfrak{L}+\frac{2 \mathfrak{L}^{2}}{\lambda}\right)\left|X_{s}^{\infty}-\widehat{X}_{s}\right|^{2}+\frac{\lambda}{2}\left|\alpha_{s}^{\infty}-\widehat{\alpha}_{s}\right|^{2},
\end{aligned}
$$


where we use the Young inequality in the last step. Now a straightforward transformation yields (45).

\section{Appendix}

Proof of Proposition 1.2. Without loss of generality we assume that $t=0$. For all $\beta \in \mathcal{A}(0 . x)$ we write $\mathcal{J}(x, \beta):=\int_{0}^{T} f\left(s, X_{s}^{x, \beta}, \beta_{s}\right) d s+g\left(X_{T}^{x, \beta}\right)$.

Let $\alpha \in \mathcal{A}(0, x)$ be optimal in (3). First, we show that $X^{\alpha}$ is non-negative. To this end define the measurable set $B:=\left\{\inf _{s \in[0, T]} X_{s}^{\alpha}<0\right\}$ and the stopping time ${ }^{2}$

$$
\sigma:=\sigma^{\alpha}:=\inf \left\{s \in[0, T] \mid X_{s}<0\right\} .
$$

Note that $B=\{\sigma<T\}$ and that $\sigma$ is equal to $\infty$ on $B^{c}$. Furthermore, $X_{\sigma}^{\alpha}=0$ on $B$. Now, define $\beta$ as the strategy which coincides with $\alpha$ on $[0, \sigma \wedge T]$, but is 0 on the interval $(\sigma \wedge T, T]$. Notice that $\alpha=\beta$ on $B^{c}$. Now let $\omega \in B$. Then $X_{s}^{\beta}(\omega)=0$ for all $s \in[\sigma(\omega), T]$, but $X_{s}^{\beta}(\omega)=X_{s}^{\alpha}(\omega)$ for $s \in[t, \sigma(\omega)]$. Also, for $s \in[\sigma(\omega), T]$,

$$
f\left(s, X_{s}^{\alpha}, \alpha_{s}\right)(\omega)-f\left(s, X_{s}^{\beta}, \beta_{s}\right)(\omega)=f\left(s, X_{s}^{\alpha}, \alpha_{s}\right)(\omega)-f(s, 0,0)(\omega) \geq 0,
$$

due to $(\mathrm{C} 1)$. Note that for $s>\sigma(\omega)$ in a sufficiently small neighbourhood of $\sigma(\omega)$ the left hand side of the previous inequality is strictly larger than zero. Moreover, (C4) yields

$$
g\left(X_{T}^{\alpha}\right)(\omega) \geq g\left(X_{T}^{\beta}\right)(\omega)=g(0)=0 .
$$

Therefore, $\mathcal{J}(x, \alpha)(\omega)>\mathcal{J}(x, \beta)(\omega)$ for all $\omega \in B$, which due to the optimality of $\alpha$ can only mean $\mathbb{P}(B)=0$. In other words $X^{\alpha}$ is non-negative a.s.

Next, we show that $X^{\alpha}$ does not have any points of increase on $[0, T]$. To this end we define the stopping times

$$
\rho:=\rho^{\alpha}:=\inf \left\{s \in[0, T] \mid X_{s}^{\alpha}>\inf _{r \in[0, s]} X_{r}^{\alpha}\right\}
$$

and

$$
\tau:=\tau^{\alpha}:=\inf \left\{s \in[0, T] \mid s>\rho \text { and } X_{s}^{\alpha}=\inf _{r \in[0, s]} X_{r}^{\alpha}\right\} .
$$

Let $\gamma$ be the strategy which is zero on $(\rho \wedge T, \tau \wedge T]$, but is equal $\alpha$ otherwise. Note that $(\rho \wedge T, \tau \wedge T]$ is empty and $\alpha=\gamma$ on

$$
\widehat{B}:=\left\{\sup _{s \in[0, T]}\left(X_{s}^{\alpha}-\inf _{r \in[0, s]} X_{r}^{\alpha}\right) \leq 0\right\},
$$

the event where $X^{\alpha}$ is non-increasing. Now let $\omega \in \widehat{B}^{c}$. Then $\rho(\omega)<T$ and $(\rho(\omega), \tau(\omega) \wedge T]$ is non-empty with $\gamma(\omega)$ vanishing on this interval and $X^{\gamma}(\omega)$ being constant $X_{\rho(\omega)}^{\gamma}(\omega)$ on this interval. Furthermore, condition (C1) implies that for all $s \in(\rho(\omega), \tau(\omega) \wedge T]$ :

$$
\begin{aligned}
f\left(s, X_{s}^{\alpha}, \alpha_{s}\right)(\omega)-f\left(s, X_{s}^{\gamma}, \gamma_{s}\right)(\omega)= & \left(f\left(s, X_{s}^{\alpha}, \alpha_{s}\right)(\omega)-f\left(s, X_{s}^{\alpha}, 0\right)(\omega)\right) \\
& +\left(f\left(s, X_{s}^{\alpha}, 0\right)(\omega)-f\left(s, X_{s}^{\gamma}, 0\right)(\omega)\right) \\
\geq & 0 .
\end{aligned}
$$

\footnotetext{
${ }^{2}$ Here and in the sequel we use the convention $\inf \emptyset=\infty$.
} 
Note that for $s>\rho(\omega)$ in a sufficiently small neighbourhood of $\rho(\omega)$ we have a strict inequality. Therefore, $\int_{0}^{T} f\left(s, X_{s}^{\alpha}, \alpha_{s}\right)(\omega) d s>\int_{0}^{T} f\left(s, X_{s}^{\gamma}, \gamma_{s}\right)(\omega) d s$. Moreover, (C4) and the convexity of $g$ imply that it is non-decreasing on the positive half-line, such that

$$
g\left(X_{T}^{\alpha}\right)(\omega) \geq g\left(X_{T}^{\gamma}\right)(\omega),
$$

and hence $\mathcal{J}(x, \alpha)(\omega)>\mathcal{J}(x, \gamma)(\omega)$. Due to optimality of $\alpha$ we have $\mathbb{P}\left(\widehat{B}^{c}\right)=0$. Therefore, the position process of an optimal strategy is non-increasing.

The second statement of the proposition can be shown with similar arguments: if $X^{\alpha}$ is not non-increasing, then we can construct $\beta$ such that $X^{\beta}$ is non-increasing and $J(0, x, \beta) \leq$ $J(0, x, \alpha)$. If in addition $X^{\beta}$ is not non-negative, then one can transform $\beta$ to $\gamma$ as above so that $X^{\gamma}$ is non-negative, non-increasing and $J(0, x, \gamma) \leq J(0, x, \beta)$.

Proof of Proposition 1.6. We adapt the proof of Theorem 5.2 in [30] to our setting and we assume again without loss of generality that $t=0$. First note that $J(0, x, \alpha)<\infty$ follows from boundedness of the processes $X$ and $\alpha$ and condition (C3). Next, let $\bar{\alpha} \in \mathcal{A}(0, x)$ and let $\bar{X}=$ $X^{0, x, \bar{\alpha}}$ be the associated state process. For the purpose of showing that $J(0, x, \bar{\alpha}) \geq J(0, x, \alpha)$ we can assume by Proposition 1.2 that $\bar{X}$ is non-increasing and non-negative, such that it is in fact bounded. Let us define $\delta X:=\bar{X}-X$ and $\delta \alpha:=\bar{\alpha}-\alpha$. Note that $\bar{X}_{0}=X_{0}=x$, such that $\delta X_{0}=0$. Also, observe that $\delta X$ is a bounded process. Since $g$ is convex we have a.s.

$$
Y_{T} \delta X_{T}-Y_{0} \delta X_{0}=Y_{T} \delta X_{T}=g^{\prime}\left(X_{T}\right) \delta X_{T} \leq g\left(\bar{X}_{T}\right)-g\left(X_{T}\right) .
$$

At the same time Itô's formula proves that

$$
\begin{aligned}
Y_{T} \delta X_{T}-Y_{0} \delta X_{0} & =\int_{0}^{T} \delta X_{s} d Y_{s}+\int_{0}^{T} Y_{s} d\left(\delta X_{s}\right) \\
& =-\int_{0}^{T}\left(\delta X_{s}\right) f_{x}\left(s, X_{s}, \alpha_{s}\right) d s+\int_{0}^{T}\left(\delta X_{s}\right) Z_{s} d W_{s}-\int_{0}^{T} Y_{s}\left(\delta \alpha_{s}\right) d s,(49)
\end{aligned}
$$

where all expressions are well-defined due to the boundedness of $X, Y$ and $\alpha=f_{y}^{*}(\cdot, X ., Y$.). Now note that for all $s \in[0, T]$

$$
\mathcal{H}_{a}\left(s, X_{s}, \alpha_{s}, Y_{s}\right)=-Y_{s}+f_{a}\left(s, X_{s}, f_{y}^{*}\left(s, X_{s}, Y_{s}\right)\right)=-Y_{s}+Y_{s}=0 .
$$

Together with the convexity of $\mathcal{H}$ this implies for all $s \in[0, T]$

$$
\begin{aligned}
\mathcal{H}\left(s, \bar{X}_{s}, \bar{\alpha}_{s}, Y_{s}\right)-\mathcal{H}\left(s, X_{s}, \alpha_{s}, Y_{s}\right) & \geq \mathcal{H}_{x}\left(s, X_{s}, \alpha_{s}, Y_{s}\right)\left(\delta X_{s}\right)+\mathcal{H}_{a}\left(s, X_{s}, \alpha_{s}, Y_{s}\right)\left(\delta \alpha_{s}\right) \\
& =f_{x}\left(s, X_{s}, \alpha_{s}\right)\left(\delta X_{s}\right) .
\end{aligned}
$$

Thus, due to the definition of $\mathcal{H}$ it holds for all $s \in[0, T]$

$$
\begin{aligned}
f\left(s, \bar{X}_{s}, \bar{\alpha}_{s}\right)-f\left(s, X_{s}, \alpha_{s}\right) & =\mathcal{H}\left(s, \bar{X}_{s}, \bar{\alpha}_{s}, Y_{s}\right)-\mathcal{H}\left(s, X_{s}, \alpha_{s}, Y_{s}\right)+Y_{s}\left(\delta \alpha_{s}\right) \\
& \geq f_{x}\left(s, X_{s}, \alpha_{s}\right)\left(\delta X_{s}\right)+Y_{s}\left(\delta \alpha_{s}\right) .
\end{aligned}
$$

This implies together with (49):

$$
Y_{T} \delta X_{T}-Y_{0} \delta X_{0} \geq \int_{0}^{T}\left(f\left(s, X_{s}, \alpha_{s}\right)-f\left(s, \bar{X}_{s}, \bar{\alpha}_{s}\right)\right) d s+\int_{0}^{T}\left(\delta X_{s}\right) Z_{s} d W_{s},
$$


where $f\left(s, X_{s}, \alpha_{s}\right)-f\left(s, \bar{X}_{s}, \bar{\alpha}_{s}\right)$, although possibly not integrable w.r.t. $s$, is in any case bounded from above and where $\int_{0}\left(\delta X_{s}\right) Z_{s} d W_{s}$ is a BMO process, such that both integrals are well defined. Combining (51) with (48) we obtain

$$
g\left(\bar{X}_{T}\right)-g\left(X_{T}\right) \geq \int_{0}^{T}\left(f\left(s, X_{s}, \alpha_{s}\right)-f\left(s, \bar{X}_{s}, \bar{\alpha}_{s}\right)\right) d s+\int_{0}^{T}\left(\delta X_{s}\right) Z_{s} d W_{s}
$$

a.s. or

$$
g\left(\bar{X}_{T}\right)+\int_{0}^{T} f\left(s, \bar{X}_{s}, \bar{\alpha}_{s}\right) d s \geq g\left(X_{T}\right)+\int_{0}^{T} f\left(s, X_{s}, \alpha_{s}\right) d s+\int_{0}^{T}\left(\delta X_{s}\right) Z_{s} d W_{s}
$$

a.s., which is true even if $\left(\int_{0}^{T} f\left(s, \bar{X}_{s}, \bar{\alpha}_{s}\right) d s\right)(\omega)=\infty$ for some $\omega$ as the right-hand-side of the inequality is a finite number a.s. Taking the expectation on both sides leads to $J(0, x, \bar{\alpha}) \geq$ $J(0, x, \alpha)$, which shows optimality of $\alpha$.

\section{References}

[1] A. Alfonsi, A. Schied, and A. Slynko. Order book resilience, price manipulation, and the positive portfolio problem. SIAM J. Financial Math., 3(1):511-533, 2012.

[2] S. Ankirchner, M. Jeanblanc, and T. Kruse. BSDEs with Singular Terminal Condition and a Control Problem with Constraints. SIAM J. Control Optim., 52(2):893-913, 2014.

[3] S. Ankirchner and T. Kruse. Optimal position targeting with stochastic linear-quadratic costs. In Advances in mathematics of finance, volume 104 of Banach Center Publ., pages 9-24. Polish Acad. Sci. Inst. Math., Warsaw, 2015.

[4] P. Bank and M. Voß. Linear quadratic stochastic control problems with singular stochastic terminal constraint. ArXiv e-prints, Nov. 2016.

[5] P. Barrieu, N. Cazanave, and N. El Karoui. Closedness results for BMO semi-martingales and application to quadratic BSDEs. C. R. Math. Acad. Sci. Paris, 346(15-16):881-886, 2008.

[6] R. Carmona and F. Delarue. Forward-backward stochastic differential equations and controlled McKean-Vlasov dynamics. Ann. Probab., 43(5):2647-2700, 2015.

[7] F. Clarke. Functional analysis, calculus of variations and optimal control, volume 264 of Graduate Texts in Mathematics. Springer, London, 2013.

[8] F. Delarue. On the existence and uniqueness of solutions to FBSDEs in a non-degenerate case. Stochastic Process. Appl., 99(2):209-286, 2002.

[9] L. C. Evans. An introduction to mathematical optimal control theory. Lecture Notes, University of California, Department of Mathematics, Berkeley, 2005.

[10] A. Fromm. Theory and applications of decoupling fields for forward-backward stochastic differential equations. PhD thesis, Humboldt-Universität zu Berlin, 2015.

[11] A. Fromm and P. Imkeller. Existence, Uniqueness and Regularity of Decoupling Fields to Multidimensional Fully Coupled FBSDEs. Preprint arXiv:1310.0499, 2013.

[12] I. M. Gelfand and S. V. Fomin. Calculus of variations. Revised English edition translated and edited by Richard A. Silverman. Prentice-Hall, Inc., Englewood Cliffs, N.J., 1963.

[13] P. Graewe, U. Horst, and J. Qiu. A non-Markovian liquidation problem and backward SPDEs with singular terminal conditions. SIAM J. Control Optim., 53(2):690-711, 2015. 
[14] Y. Hu and S. Peng. Solution of forward-backward stochastic differential equations. Probab. Theory Relat. Fields, 103(2):273-283, 1995.

[15] T. Kruse. Inverse Optimal Stopping and Optimal Closure of Illiquid Positions. PhD thesis, Universitäts-und Landesbibliothek Bonn, 2014.

[16] T. Kruse and A. Popier. Minimal supersolutions for BSDEs with singular terminal condition and application to optimal position targeting. Stochastic Process. Appl., 126(9):2554-2592, 2016.

[17] J. Ma, P. Protter, and J. Yong. Solving forward-backward stochastic differential equations explicitly - a four step scheme. Probab. Theory Relat. Fields, 98(3):339-359, 1994.

[18] J. Ma, Z. Wu, D. Zhang, and J. Zhang. On well-posedness of forward-backward SDEs - a unified approach. Ann. Appl. Probab., 25(4):2168-2214, 2015.

[19] J. Ma, H. Yin, and J. Zhang. On non-Markovian forward-backward SDEs and backward stochastic PDEs. Stochastic Process. Appl., 122(12):3980-4004, 2012.

[20] J. Ma and J. Yong. Forward-backward stochastic differential equations and their applications, volume 1702 of Lecture Notes in Mathematics. Springer-Verlag, Berlin, 1999.

[21] J. Ma and J. Yong. On linear, degenerate backward stochastic partial differential equations. Probab. Theory Related Fields, 113(2):135-170, 1999.

[22] T. Mikami and M. Thieullen. Optimal transportation problem by stochastic optimal control. SIAM J. Control Optim., 47(3):1127-1139, 2008.

[23] E. Pardoux and S. Tang. Forward-backward stochastic differential equations and quasilinear parabolic PDEs. Probab. Theory Relat. Fields, 114(2):123-150, 1999.

[24] S. Peng and Z. Wu. Fully coupled forward-backward stochastic differential equations and applications to optimal control. SIAM J. Control Optim., 37(3):825-843, 1999.

[25] A. Popier. Backward stochastic differential equations with singular terminal condition. Stochastic Process. Appl., 116(12):2014-2056, 2006.

[26] A. Popier. Backward stochastic differential equations with random stopping time and singular final condition. Ann. Probab., 35(3):1071-1117, 2007.

[27] X. Tan and N. Touzi. Optimal transportation under controlled stochastic dynamics. Ann. Probab., 41(5):3201-3240, 2013.

[28] J. Yong. Finding adapted solutions of forward-backward stochastic differential equations: Method of continuation. Probab. Theory Relat. Fields, 107(4):537-572, 1997.

[29] J. Yong. Forward-backward stochastic differential equations with mixed initial-terminal conditions. Trans. Amer. Math. Soc., 362(2):1047-1096, 2010.

[30] J. Yong and X. Y. Zhou. Stochastic controls, volume 43 of Applications of Mathematics (New York). Springer-Verlag, New York, 1999. Hamiltonian systems and HJB equations.

[31] J. Yong and X. Y. Zhou. Stochastic controls: Hamiltonian systems and HJB equations. Springer, 1999.

[32] W. P. Ziemer. Weakly differentiable functions, volume 120 of Graduate Texts in Mathematics. Springer-Verlag, New York, 1989. Sobolev spaces and functions of bounded variation. 